\title{
Inf-Sup Conditions for Twofold Saddle Point Problems
}

\author{
Noel J. Walkington ${ }^{1 \star}$, Jason S. Howell ${ }^{2 \star \star}$ \\ 1 Department of Mathematical Sciences, Carnegie Mellon University, Pittsburgh, PA 15213, e-mail: noelw@andrew . cmu.edu \\ 2 Department of Mathematical Sciences, Carnegie Mellon University, Pittsburgh, PA 15213, e-mail: howell4@andrew. cmu . edu
}

Submitted to Numerische Mathematik: June 17, 2009

Summary Necessary and sufficient conditions for existence and uniqueness of solutions are developed for twofold saddle point problems which arise in mixed formulations of problems in continuum mechanics. This work extends the classical saddle point theory to accommodate nonlinear constitutive relations and the twofold saddle structure. Application to problems in incompressible fluid mechanics employing symmetric tensor finite elements for the stress approximation is presented.

Key words saddle point problem - twofold saddle point problem - inf-sup condition - ArnoldWinther element

Mathematics Subject Classification (1991): $65 \mathrm{~N} 30$

\section{Introduction}

Many problems in continuous fluid and solid mechanics lead to a variational problems with a saddle point structure of the form: $\left(u, p_{1}, p_{2}\right) \in U \times P_{1} \times P_{2}$,

$$
\left[\begin{array}{ccc}
A & -B_{1}^{T} & -B_{2}^{T} \\
B_{1} & 0 & 0 \\
B_{2} & 0 & 0
\end{array}\right]\left[\begin{array}{c}
u \\
p_{1} \\
p_{2}
\end{array}\right]=\left[\begin{array}{c}
f \\
g_{1} \\
g_{2}
\end{array}\right]
$$

or

$$
\left[\begin{array}{ccc}
A & 0 & -B_{1}^{T} \\
0 & 0 & -B_{2}^{T} \\
B_{1} & B_{2} & 0
\end{array}\right]\left[\begin{array}{c}
u \\
p_{1} \\
p_{2}
\end{array}\right]=\left[\begin{array}{c}
f \\
g_{1} \\
g_{2}
\end{array}\right] .
$$

Here $U, P_{1}$ and $P_{2}$ are Banach spaces, $A: U \rightarrow U^{\prime}$ is typically nonlinear, and $B_{1}$ and $B_{2}$ are linear operators. In this paper we develop necessary and sufficient conditions for this class of problems to be well-posed and consider their numerical approximation.

\footnotetext{
* Supported in part by National Science Foundation Grants DMS-0811029. This work was also supported by the NSF through the Center for Nonlinear Analysis.

** This material is based upon work supported by the Center for Nonlinear Analysis (CNA) under the National Science Foundation Grant No. DMS-0635983.
} 
Problems of type (1.2) can be written as

$$
\left[\begin{array}{ccc}
A & -B_{1}^{T} & 0 \\
B_{1} & 0 & B_{2} \\
0 & -B_{2}^{T} & 0
\end{array}\right]\left[\begin{array}{c}
u \\
p_{2} \\
p_{1}
\end{array}\right]=\left[\begin{array}{c}
f \\
g_{2} \\
g_{1}
\end{array}\right] .
$$

These problems are often described as twofold saddle point problems due to the nested structure they exhibit. Each of these problems can be viewed as a single saddle point problem of the form $\left[\begin{array}{cc}A & -B^{T} \\ B & 0\end{array}\right]=\left[\begin{array}{l}f \\ g\end{array}\right]$ where $B$ acts on a product space. The classical theory requires $B$ to satisfy an infsup condition $[8,13,14]$. The development of discrete spaces which inherit such inf-sup conditions for each specific problem is notoriously difficult, and this difficulty is compounded by the product structure of the twofold saddle point problems. In Lemmas 3.1 and 3.2 we establish necessary and sufficient inf-sup conditions on the constituent operators $B_{1}$ and $B_{2}$ to guarantee that the compound operator $B$ satisfies inf-sup conditions on the corresponding product space.

The rest of this paper is organized as follows. This introductory section continues with a motivating example, followed by a review of prior results and some notation. Section 2 considers solution of single saddle point problems with nonlinear operator $A$ and their Galerkin approximation. Section 3 develops necessary and sufficient conditions on the operators $B_{1}$ and $B_{2}$ for problems of type (1.1) and (1.2) to be well posed. An application of the theory is illustrated in Section 4.

\subsection{Examples of Twofold Saddle Point Problems}

Twofold saddle point problems arise ubiquitously when mixed finite element formulations are used to approximate the stress in an incompressible fluid or solid $[2,9,27,29,24,15,22,23,6,26]$. When the usual linear relation $S_{0}=\nu D(\mathbf{u})$ for the viscous stress is replaced with a more general relation $S_{0}=\nu \mathcal{A}(D(\mathbf{u}))$ the equations for the creeping (Stokes) flow of a fluid in a domain $\Omega \subset \mathbb{R}^{d}$ become,

$$
-\operatorname{div}(S)=\mathbf{f}, \quad \operatorname{div}(\mathbf{u})=0, \quad S=-p I+\mathcal{A}(D(\mathbf{u})) .
$$

Here $D(\mathbf{u})=(1 / 2)\left(\nabla \mathbf{u}+(\nabla \mathbf{u})^{T}\right), S$ is the stress tensor, and for incompressible fluids takes the form $S=-p I+S_{0}$ where $\operatorname{tr}\left(S_{0}\right)=0$ is the devatoric part of the stress. If $\partial \Omega=\Gamma_{0} \cup \Gamma_{1}$ typical boundary conditions would be

$$
\left.\mathbf{u}\right|_{\Gamma_{0}}=u_{0},\left.\quad S n\right|_{\Gamma_{1}}=\mathbf{t} .
$$

To illustrate our results we review three formulations of this problem:

(1) A single saddle point problem arises when the stress $S$ is eliminated using the constitutive relation as in [30] (classical primal mixed formulation).

(2) Eliminating the gradient of the velocity using the constitutive relation gives rise to a dual mixed variational formulation [32]. A twofold saddle point problem of the form (1.1) then arises when the problem is posed in three variables [9] or the symmetry of the stress tensor is enforced weakly $[2]$.

(3) Alternatively, independent approximation of (the symmetric part of) the velocity gradient gives rise to a twofold saddle problem of the form (1.2) (alternate dual mixed formulation). Dual mixed approaches that approximate the (nonsymmetric) velocity gradient directly are presented in [24, $19]$.

When the fluid is incompressible the stress-strain relation only acts on the trace free (devatoric) part of the strain. Letting $\left(\mathbb{R}_{\text {sym }}^{d \times d}\right)_{0}$ denote the symmetric trace free matrices, we assume

1. $\mathcal{A}: \mathbb{R}^{d \times d} \rightarrow\left(\mathbb{R}_{\text {sym }}^{d \times d}\right)_{0}$. 
2. $\mathcal{A}(D)=\mathcal{A}\left(D_{\text {sym }}-(\operatorname{tr}(D) / d) I\right)$.

3. The restriction $\mathcal{A}:\left(\mathbb{R}_{\text {sym }}^{d \times d}\right)_{0} \rightarrow\left(\mathbb{R}_{\text {sym }}^{d \times d}\right)_{0}$ is bijective.

Primal Mixed Formulation: The classical weak statement of this problem seeks $\mathbf{u}-u_{0} \in U=$ $\left\{\mathbf{u} \in H^{1}(\Omega)^{d}|\mathbf{u}|_{\Gamma_{0}}=0\right\}$ and $p \in L^{2}(\Omega)$ satisfying

$$
\begin{gathered}
\int_{\Omega}(\mathcal{A}(D(\mathbf{u})): D(\mathbf{v})-p \operatorname{div}(\mathbf{v}))=\int_{\Omega} \mathbf{f} . \mathbf{v}+\int_{\Gamma_{1}} \mathbf{t . v}, \quad \mathbf{v} \in U, \\
\int_{\Omega} \operatorname{div}(\mathbf{u}) q=0, \quad q \in L^{2}(\Omega) .
\end{gathered}
$$

If $\Gamma_{1}=\emptyset$, then $p, q$ are required to have average zero. This is a classical saddle point problem taking the form

$$
\left[\begin{array}{cc}
\mathcal{A} & -\operatorname{div}^{\prime} \\
\operatorname{div} & 0
\end{array}\right]\left[\begin{array}{l}
\mathbf{u} \\
p
\end{array}\right]=\left[\begin{array}{c}
\mathbf{f}+\gamma^{\prime}(\mathbf{t}) \\
0
\end{array}\right]
$$

Here $\left\langle\operatorname{div}^{\prime}(p), \mathbf{u}\right\rangle=(p, \operatorname{div}(\mathbf{u}))$ is the dual operator, and similarly $\left\langle\gamma^{\prime}(\mathbf{t}), \mathbf{v}\right\rangle=(\mathbf{t}, \gamma(\mathbf{v}))_{\Gamma_{1}}$ is the dual of the trace operator $\gamma: U \rightarrow U / H_{0}^{1}(\Omega)^{d}$. Here $(\cdot, \cdot)$ is the $L^{2}(\Omega)$ inner product and $\langle\cdot, \cdot\rangle$ is the induced duality pairing.

Dual Mixed Formulation: If the inverse of the stress strain relation is available, $D(\mathbf{u})=$ $\mathcal{A}^{-1}(S)$, it is possible to write a weak statement that evaluates the stress explicitly. Let

$$
\mathbb{S}=\left\{S \in \mathbb{H}(\Omega ; \operatorname{div})_{s y m}|S \mathbf{n}|_{\Gamma_{1}}=0\right\} \quad \text { and } \quad U=L^{2}(\Omega),
$$

and $\mathbb{S}(\mathbf{t})$ be the elements in $S \in \mathbb{H}(\Omega ; \text { div })_{\text {sym }}$ for which $\left.S \mathbf{n}\right|_{\Gamma_{1}}=\mathbf{t}$. Then $(S, \mathbf{u}) \in \mathbb{S}(\mathbf{t}) \times U$ satisfies

$$
\begin{gathered}
\int_{\Omega}\left(\mathcal{A}^{-1}(S): T+\mathbf{u} \cdot \operatorname{div}(T)\right)=\int_{\Gamma_{0}} \mathbf{u}_{0} \cdot T \mathbf{n}, \quad T \in \mathbb{S}, \\
\int_{\Omega}-\operatorname{div}(S) \cdot \mathbf{v}=\int_{\Omega} f \cdot \mathbf{v} . \quad \mathbf{v} \in U .
\end{gathered}
$$

This weak statement is again a classical saddle point problem. A mixed finite element method for this formulation in linear elasticity was studied in [3].

If finite element subspaces of $\mathbb{H}(\Omega \text {; div })_{\text {sym }}$ are not available, it is possible to pose the previous weak statement in $\mathbb{H}(\Omega ; \operatorname{div})$ and to use Raviart-Thomas elements. In this instance $\nabla \mathbf{u}=\mathcal{A}^{-1}(S)+$ $(\nabla \mathbf{u})_{\text {skew. }}$. Let

$$
\mathbb{T}=\left\{S \in \mathbb{H}(\Omega ; \operatorname{div})|S \mathbf{n}|_{\Gamma_{1}}=0\right\} \quad \text { and } \quad U=\left(L^{2}(\Omega)\right)^{2},
$$

and $\mathbb{T}(\mathbf{t})$ be the elements in $S \in \mathbb{H}(\Omega ; \operatorname{div})$ for which $\left.S \mathbf{n}\right|_{\Gamma_{1}}=\mathbf{t}$. Next, define $W: \mathbb{R}^{d} \rightarrow \mathbb{R}_{\text {skew }}^{d \times d}$ by $W(\mathbf{w})_{i j}=\epsilon_{i j k} w_{k}$ Then $(S, \mathbf{u}, \mathbf{w}) \in \mathbb{T}(\mathbf{t}) \times U \times L^{2}(\Omega)$ satisfies

$$
\begin{gathered}
\int_{\Omega}\left(\mathcal{A}^{-1}(S): T+W(\mathbf{w}): T+\mathbf{u} \cdot \operatorname{div}(T)\right)=\int_{\Gamma_{0}} \mathbf{u}_{0} \cdot T \mathbf{n}, \quad T \in \mathbb{T}, \\
\int_{\Omega}-\operatorname{div}(S) \cdot \mathbf{v}=\int_{\Omega} f \cdot \mathbf{v}, \quad \mathbf{v} \in U \\
\int_{\Omega}-S: W(\mathbf{z})=0, \quad \mathbf{z} \in L^{2}(\Omega) .
\end{gathered}
$$

This is a twofold saddle point problem taking the form

$$
\left[\begin{array}{ccc}
\mathcal{A}^{-1} & \operatorname{div}^{\prime} & W \\
-\operatorname{div} & 0 & 0 \\
-W^{\prime} & 0 & 0
\end{array}\right]\left[\begin{array}{c}
S \\
\mathbf{u} \\
\mathbf{w}
\end{array}\right]=\left[\begin{array}{c}
\gamma^{\prime}\left(\mathbf{u}_{0}\right) \\
\mathbf{f} \\
0
\end{array}\right] .
$$


Here $\left\langle\gamma^{\prime}\left(\mathbf{u}_{0}\right), T\right\rangle=\left(\mathbf{u}_{0}, T \mathbf{n}\right)_{\Gamma_{0}}$.

Finite element methods for the formulation (1.4) arising in linear elasticity were developed by Arnold, Brezzi, and Douglas [2] (see [6] for a survey of approaches with weakly imposed symmetry).

Alternative Dual Mixed Formulation: If the inverse $\mathcal{A}^{-1}$ if the constitutive relation is not available it is possible to pose a mixed formulation which computes both $D(\mathbf{u})$ and $S$. Let $\mathbb{S}$ and $U=L^{2}(\Omega)^{d}$ be as above and

$$
\mathbb{D}=\left\{D \in L^{2}(\Omega)_{\text {sym }}^{d \times d} \mid \operatorname{tr}(D)=0\right\} .
$$

and $\mathbb{S}(\mathbf{t})$ be the elements in $S \in \mathbb{H}(\Omega ; \operatorname{div})_{\text {sym }}$ for which $\left.S \mathbf{n}\right|_{\Gamma_{1}}=\mathbf{t}$. Then $(D, \mathbf{u}, S) \in \mathbb{D} \times U \times \mathbb{S}(\mathbf{t})$ satisfies

$$
\begin{gathered}
\int_{\Omega} \mathcal{A}(D): E-S: E=0, \quad E \in \mathbb{D} \\
\int_{\Omega}(D: T+\mathbf{u} \cdot \operatorname{div}(T))=\int_{\Gamma_{0}} \mathbf{u}_{0} \cdot T \mathbf{n}, \quad T \in \mathbb{S}, \\
\int_{\Omega}-\operatorname{div}(S) \cdot \mathbf{v}=\int_{\Omega} f \cdot \mathbf{v} . \quad \mathbf{v} \in U .
\end{gathered}
$$

This is a twofold saddle point problem taking the form

$$
\left[\begin{array}{ccc}
\mathcal{A} & 0 & -I \\
0 & 0 & -\operatorname{div} \\
I^{\prime} & \operatorname{div}^{\prime} & 0
\end{array}\right]\left[\begin{array}{l}
D \\
\mathbf{u} \\
S
\end{array}\right]=\left[\begin{array}{c}
0 \\
\mathbf{f} \\
\gamma^{\prime}\left(\mathbf{u}_{0}\right)
\end{array}\right] .
$$

If the trace-free requirement on tensors in $\mathbb{D}$ is relaxed a formulation which includes the pressure requires $(D, \mathbf{u}, S, p) \in L^{2}(\Omega)_{\text {sym }}^{d \times d} \times U \times \mathbb{S}(\mathbf{t}) \times P$ satisfying

$$
\begin{aligned}
\int_{\Omega} \mathcal{A}(D): E-S: E-p I: E & =0, \quad E \in L^{2}(\Omega)_{\text {sym }}^{d \times d}, \\
\int_{\Omega}(D: T+q I: D+\mathbf{u} \cdot \operatorname{div}(T)) & =\int_{\Gamma_{0}} \mathbf{u}_{0} \cdot T \mathbf{n}, \quad(T, q) \in \mathbb{S} \times P, \\
\int_{\Omega}-\operatorname{div}(S) \cdot \mathbf{v} & =\int_{\Omega} f \cdot \mathbf{v} . \quad \mathbf{v} \in U .
\end{aligned}
$$

where $P=L^{2}(\Omega)$. This formulation takes the form of (1.5) where the identity operator is replaced with $I \times p \operatorname{tr}($.$) .$

\subsection{Related Results}

Problems (1.1) and (1.2) are simplified versions of a full twofold saddle point problem described by Brezzi and Fortin [14, pp. 41, §II.1]. Existing results regarding different formulations of inf-sup conditions for problems of type (1.1) can be found in [28] and [20]. Problems with this structure arise in various applications, including elasticity $([2,6])$, complex fluids $([9,20])$, and hybridized mixed methods for second order elliptic problems [18]. Solvability of these problems is usually established by showing that $A$ has the required properties and either (i) the combined operator $B_{1}+B_{2}$ is surjective or (ii) the operators $B_{1}$ and $B_{2}$ individually satisfy appropriate inf-sup conditions.

Problems of type (1.2) are analyzed in a similar manner. In [21] and [25], problems of this type were treated as nested saddle problems, with the upper left $2 \times 2$ block of (1.3) being treated as one operator and then showing that block is coercive over the kernel of the operator $B_{2}$. Sufficient 
conditions for solvability and abstract error analysis are also given in [21] and [25]. This theory has been applied to many problems in elasticity and fluids as well, see $[27,29,24,15,22,23,26]$ for some examples. Problems of type $(1.2)$ were also studied in $[16,17]$. In these works the twofold saddle point problems are treated as single saddle point problems, and solvability is shown by requiring $B_{1}+B_{2}$ to satisfy a certain inf-sup condition.

\subsection{Notation}

For Banach spaces $X$ and $Y, X^{\prime}$ and $Y^{\prime}$ denote their duals, and if $F: X \rightarrow Y$ is an operator, its dual operator is denoted by $F^{\prime}: Y^{\prime} \rightarrow X^{\prime}$ or $F^{T}: Y^{\prime} \rightarrow X^{\prime}$. If $Z \subset X$ is a closed subspace the quotient space is denoted by $X / Z$ and frequently $x \in X$ is identified with $x+Z \in X / Z$. The dual space $(X / Z)^{\prime}$ will be identified with the (polar) subspace of $X^{\prime}$,

$$
Z^{0}=\left\{f \in X^{\prime} \mid f(z)=0, z \in Z\right\} .
$$

Under this identification $\|f\|_{(X / Z)^{\prime}}=\|f\|_{X^{\prime}}$.

Standard notation is used for the Lebesgue spaces $L^{p}(\Omega)$, the Sobolev spaces $W^{k, p}(\Omega)$ and $H^{k}(\Omega)=W^{k, 2}(\Omega)$. We will always assume $\Omega \subset \mathbb{R}^{d}$ is a bounded Lipschitz domain with boundary partition $\partial \Omega=\Gamma_{0} \cup \Gamma_{1}$. The inner product $L^{2}(\Omega)$ will be denoted as $(\cdot, \cdot)$. Recall that $H(\Omega$; div $)$ is the space of vector valued functions in $L^{2}(\Omega)^{d}$ having divergence in $L^{2}(\Omega)$,

$$
H(\Omega ; \operatorname{div})=\left\{v \in L^{2}(\Omega)^{d} \mid \operatorname{div}(v) \in L^{2}(\Omega)\right\},
$$

equipped with inner-product

$$
(v, w)_{H(\Omega ; \operatorname{div})}=(v, w)+(\operatorname{div}(v), \operatorname{div}(w)) .
$$

The space of matrix (tensor) valued functions $S \in L^{2}(\Omega)^{d \times d}$ having divergence $\operatorname{div}(S)=S_{i j . j} \in$ $L^{2}(\Omega)^{d}$ is denoted by $\mathbb{H}(\Omega ; \operatorname{div})$ with inner product

$$
(S, T)_{\mathbb{H}(\Omega ; \operatorname{div})}=(S, T)+(\operatorname{div}(S), \operatorname{div}(T)) .
$$

Matrix valued quantities will be denoted with upper case letters, vectors with bold face lower case letters.

The Generalized Lax Milgram theorem will be used ubiquitously below. The following is a particularly convenient statement of this fundamental result.

Theorem 1.1 (Generalized Lax Milgram) Let $U$ and $V$ be a Banach spaces, $V$ be reflexive, and $c>0$. Let $a: U \times V \rightarrow \mathbb{R}$ be bilinear and continuous; that is, there exists $C>0$ such that $|a(u, v)| \leq C\|u\|_{U}\|v\|_{V}$ for all $u \in U$ and $v \in V$. Then the following are equivalent:

[C] (Coercivity) For each $u \in U$,

$$
\sup _{0 \neq v \in V} \frac{a(u, v)}{\|v\|_{V}} \geq c\|u\|_{U},
$$

and for each $v \in V \backslash\{0\}, \sup _{u \in U} a(u, v)>0$.

[E] (Existence of Solutions) For each $f \in V^{\prime}$ there exists a unique $u \in U$ such that

$$
a(u, v)=f(v), \quad \forall v \in V,
$$

and $\|u\|_{U} \leq(1 / c)\|f\|_{V^{\prime}}$. 
$\left[E^{\prime}\right]$ (Existence of Solutions for the Adjoint Problem) For each $g \in U^{\prime}$ there exists a unique $v \in V$ such that

$$
a(u, v)=g(u), \quad \forall u \in U
$$

and $\|v\|_{V} \leq(1 / c)\|g\|_{U^{\prime}}$.

In addition, if $U$ is reflexive then each of the above are equivalent to:

$\left[C^{\prime}\right]$ (Adjoint Coercivity) For each $u \in U$,

$$
\sup _{0 \neq u \in U} \frac{a(u, v)}{\|u\|_{U}} \geq c\|v\|_{V}
$$

and for each $u \in U \backslash\{0\}, \sup _{v \in V} a(u, v)>0$.

The following classical result for linear saddle point problems is a special case of this theorem.

Corollary 1.1 Let $U$ and $P$ be reflexive Banach spaces and assume that $a: U \times U \rightarrow \mathbb{R}$ and $b: P \times U \rightarrow \mathbb{R}$ are continuous and bilinear. Then the following are equivalent.

1. (Existence of Solutions) For all $f \in U^{\prime}$ and $g \in P^{\prime}$ there exists $(u, p) \in U \times P$ such that

$$
a(u, v)-b(p, v)=f(v), \quad \text { and } \quad b(q, u)=g(q),
$$

for all $(v, q) \in U \times P$, and there exists $C>0$ such that $\|u\|_{U}+\|p\|_{P} \leq C\left(\|f\|_{U^{\prime}}+\|g\|_{P^{\prime}}\right)$.

2. There exists a constant $c>0$ such that

- The restriction $a: Z \times Z \rightarrow \mathbb{R}$ is coercive over $Z \equiv\{u \in U \mid b(p, u)=0, \forall p \in P\}$.

$$
\sup _{v \in Z} \frac{a(u, v)}{\|v\|_{U}} \geq c\|u\|_{U}, \quad \text { and } \quad \sup _{u \in Z} a(u, v)>0, \quad \text { for all } 0 \neq v \in Z
$$

- (inf-sup condition) $\sup _{u \in U} \frac{b(p, u)}{\|u\|_{U}} \geq c\|p\|_{P}$.

3. (Coercivity) The bilinear map $A((u, p),(v, q))=a(u, v)-b(p, v)+b(q, u)$, is coercive on $U \times P$ : there exists $c>0$ such that

$$
\sup _{(v, q) \in U \times P} \frac{A((u, p),(v, q))}{\|v\|_{U}+\|q\|_{P}} \geq c\left(\|u\|_{U}+\|p\|_{P}\right)
$$

and

$$
\sup _{(u, p) \in U \times P} A((u, p),(v, q))>0, \quad \text { for all }(0,0) \neq(v, q) \in U \times P .
$$

If $b: U \times P \rightarrow \mathbb{R}$ satisfies the inf-sup condition in (2) and $Z$ is the kernel, then the restriction $b: U / Z \times P \rightarrow \mathbb{R}$ satisfies coercivity hypothesis [C] of the Lax Milgram theorem. We make frequent use of the fact that, in this situation, this implies adjoint coercivity $\left[\mathrm{C}^{\prime}\right]$ as well as existence for both the primal and dual problem. 


\section{Nonlinear Saddle Point Problem}

Viscoelastic properties of fluids are frequently modeled with a nonlinear constitutive relation. When the fluid is incompressible this gives rise to a saddle point problem of the form; $(u, p) \in U \times P$,

$$
\begin{aligned}
a(u, v)-b(p, v) & =f(v), \\
b(q, u) & =g(q),
\end{aligned}
$$

for all $(v, q) \in U \times P$, where $a: U \times U \rightarrow \mathbb{R}$ is linear in the second argument and $b: U \times P \rightarrow \mathbb{R}$ is bilinear. When $a(.,$.$) is bilinear the Lax Milgram theorem shows that this problem is well-posed if$ (and only if) $b(.,$.$) satisfies and inf-sup condition and a(.,$.$) is coercive over the kernel of b(.,$.$) . The$ following theorem is the natural extension of this result to the situation where $a(.,$.$) is nonlinear.$

Theorem 2.1 Let $U$ and $P$ be reflexive Banach spaces, $b: P \times U \rightarrow \mathbb{R}$ be continuous and bilinear, and let $a: U \times U \rightarrow \mathbb{R}$ be linear in its second argument. Let $Z=\{u \in U \mid b(p, u)=0, \forall p \in P\}$ and $c>0$. Then the following are equivalent.

1. (Existence of Solutions) For all $f \in U^{\prime}$ and $g \in P^{\prime}$ there exists $(u, p) \in U \times P$ such that (a)

$$
a(u, v)-b(p, v)=f(v), \quad \text { and } \quad b(q, u)=g(q),
$$

for all $(v, q) \in U \times P$, and $(b)\|u\|_{U / Z} \leq(1 / c)\|g\|_{P^{\prime}}$.

2. (a) For all $f \in U^{\prime}$ and $u_{g} \in U$ there exists $u \in U$ such that $u-u_{g} \in Z$ and

$$
a(u, v)=f(v), \quad v \in Z,
$$

(b) (inf-sup condition)

$$
\sup _{u \in U} \frac{b(p, u)}{\|u\|_{U}} \geq c\|p\|_{P}
$$

Proof When $b(.,$.$) is viewed as a bilinear form on P \times U / Z$ the equivalence of statements $1(\mathrm{~b})$ and 2(b) follow directly from the Generalized Lax Milgram theorem.

$(1) \Rightarrow(2):$ Fix $f \in U^{\prime}, u_{g} \in U$ and let $(u, p) \in U \times P$ satisfying

$$
a(u, v)-b(p, v)=f(v), \quad b(q, u)=b\left(q, u_{g}\right), \quad(v, q) \in U \times P,
$$

with $\|u\|_{U / Z} \leq\left(C_{b} / c\right)\left\|u_{g}\right\|_{U / Z}$. Then $u-u_{g} \in Z$ so $u$ satisfies 2(a).

$(2) \Rightarrow(1):$ Fix $(f, g) \in U^{\prime} \times P^{\prime}$. The Generalized Lax Milgram applied to $b: P \times U / Z \rightarrow \mathbb{R}$ guarantees the existence of $u_{g} \in U$ such that $b\left(q, u_{g}\right)=g(q)$ and $\left\|u_{g}\right\|_{U / Z} \leq(1 / c)\|g\|_{P^{\prime}}$. Let $u \in U$ satisfy $u-u_{g} \in Z$ and $a(u, v)=f(v)$ for $v \in Z$. Then $b(u, q)=g(q)$,

$$
\|u\|_{U / Z}=\left\|u_{g}\right\|_{U / Z} \leq(1 / c)\|g\|_{P^{\prime}}
$$

and $a(u, v)-f(v)=0$ for $v \in Z$; that is, $a(u,)-.f(.) \in(U / Z)^{\prime}$. The Generalized Lax Milgram theorem applied to $b: P \times U / Z \rightarrow \mathbb{R}$ then guarantees the existence of $p \in P$ such that

$$
a(u, v)-f(v)=b(p, v), \quad v \in U,
$$

which establishes $1(\mathrm{a})$.

\section{Remarks:}

1. Uniqueness of solutions will follow if, for example,

$$
u_{2}-u_{1} \in Z \text { and } a\left(u_{2}, v\right)-a\left(u_{1}, v\right)=0, v \in Z \quad \Rightarrow \quad u_{2}=u_{1} .
$$

2. The above theorem is valid if $a: D(A) \times U \rightarrow \mathbb{R}$ has domain strictly contained in $U$ provided the hypotheses in (1) and (2) require $u \in D(A)$. 


\subsection{Finite Element Approximation}

We consider Galerkin approximations of solutions to the saddle point problems in Theorem 2.1. Approximation properties of Galerkin schemes for nonlinear problems depend in a non-trivial fashion upon the structure of the nonlinearity, $[10,11]$. In this section linearity of the constraints is exploited to develop some useful formulae for the error. These are then used to develop estimates for the special case where the nonlinear operator is strictly monotone and Lipschitz.

Lemma 2.1 Let $U$ and $P$ be reflexive Banach spaces, $b: P \times U \rightarrow \mathbb{R}$ be continuous and bilinear, and let $a: U \times U \rightarrow \mathbb{R}$ be linear in its second argument. Let $Z=\{u \in U \mid b(p, u)=0, \forall p \in P\}$ and assume that $b(.,$.$) satisfies the inf-sup condition: there exists c>0$ such that

$$
\sup _{v \in U} \frac{b(p, v)}{\|v\|_{U}} \geq c\|p\|_{P}
$$

Fix $(f, g) \in U^{\prime} \times P^{\prime}$ and suppose that $(u, p) \in U \times P$ satisfies

$$
a(u, v)-b(p, v)=f(v), \quad b(q, u)=g(q), \quad(v, q) \in U \times P .
$$

Let $U_{h} \subset U$ and $P_{h} \subset P$ be subspaces and suppose $\left(u_{h}, p_{h}\right) \in U_{h} \times P_{h}$ satisfy

$$
a\left(u_{h}, v_{h}\right)-b\left(p_{h}, v_{h}\right)=f\left(v_{h}\right), \quad b\left(q_{h}, u_{h}\right)=g\left(q_{h}\right), \quad\left(v_{h}, q_{h}\right) \in U_{h} \times P_{h} .
$$

Then

$$
a\left(u, v_{h}\right)-a\left(u_{h}, v_{h}\right)=b\left(p-q_{h}, v_{h}\right), \quad\left(v_{h}, q_{h}\right) \in Z_{h} \times P_{h},
$$

and

$$
\left\|p-p_{h}\right\|_{P} \leq(1+C / c)\left\|p-q_{h}\right\|_{P}+(1 / c) \sup _{v_{h} \in U_{h}} \frac{a\left(u, v_{h}\right)-a\left(u_{h}, v_{h}\right)}{\left\|v_{h}\right\|_{U}}, \quad q_{h} \in P_{h},
$$

where $Z_{h}=\left\{u_{h} \in U_{h} \mid b\left(p_{h}, u_{h}\right)=0, \forall p_{h} \in P_{h}\right\}$ is the discrete null space of $b(.,$.$) .$

Proof The Galerkin orthogonality relations for this problem becomes

$$
a\left(u, v_{h}\right)-a\left(u_{h}, v_{h}\right)-b\left(p-p_{h}, v_{h}\right)=0, \quad v_{h} \in U_{h},
$$

and (this condition is not used here, see the following lemma)

$$
b\left(q_{h}, u-u_{h}\right)=0, \quad q_{h} \in P_{h} .
$$

Restricting the test functions in the first Galerkin orthogonality statement to to $v_{h} \in Z_{h}$ shows

$$
a\left(u, v_{h}\right)-a\left(u_{h}, v_{h}\right)=b\left(p-q_{h}, v_{h}\right), \quad\left(v_{h}, q_{h}\right) \in Z_{h} \times P_{h} .
$$

Using the inf-sup condition on $b(.,$.$) we find$

$$
\begin{aligned}
\left\|p-p_{h}\right\|_{P} & \leq\left\|p-q_{h}\right\|_{P}+\left\|q_{h}-p_{h}\right\|_{P} \\
& \leq\left\|p-q_{h}\right\|_{P}+(1 / c) \sup _{v_{h} \in U_{h}} \frac{b\left(q_{h}-p_{h}, v_{h}\right)}{\left\|v_{h}\right\|_{U}} \\
& \leq\left\|p-q_{h}\right\|_{P}+(1 / c) \sup _{v_{h} \in U_{h}} \frac{b\left(q_{h}-p+p-p_{h}, v_{h}\right)}{\left\|v_{h}\right\|_{U}} \\
& \leq(1+C / c)\left\|p-q_{h}\right\|_{P}+(1 / c) \sup _{v_{h} \in U_{h}} \frac{a\left(u, v_{h}\right)-a\left(u_{h}, v_{h}\right)}{\left\|v_{h}\right\|_{U}}
\end{aligned}
$$


The first statement of this lemma typically gives rise to estimates of the form $\left\|u-u_{h}\right\|_{U} \leq$ $C\left(\left\|u-v_{h}\right\|_{U}+\left\|p-p_{h}\right\|_{P}\right)$ for all $\left(v_{h}, q_{h}\right) \in Z_{h} \times P_{h}$. The proof of this lemma did not use the Galerkin orthogonality relation (2.5) arising from the constraint equation. This relation is used to show that $u-u_{h}$ satisfies the hypotheses of the following classical result which shows that approximation of $u$ by $v_{h} \in u_{h}+Z_{h}$ is optimal.

Lemma 2.2 Let $U$ and $P$ be reflexive Banach spaces and $b: P \times U \rightarrow \mathbb{R}$ be continuous and bilinear, and satisfy the inf-sup condition

$$
\sup _{u \in U} \frac{b(p, u)}{\|u\|} \geq c_{b}\|p\|, \quad u \in U
$$

Let $U_{h} \subset U$ and $P_{h} \subset P$ be subspaces and let

$$
\hat{Z}_{h}=\left\{u \in U \mid b\left(p_{h}, u\right)=0, p_{h} \in P_{h}\right\}
$$

and

$$
Z_{h}=U_{h} \cap \hat{Z}_{h}=\left\{u_{h} \in U_{h} \mid b\left(p_{h}, u_{h}\right)=0, p_{h} \in P_{h}\right\} .
$$

Then the following are equivalent.

1. The restriction of $b$ to $U_{h} \times P_{h}$ satisfies the inf-sup condition,

$$
\sup _{u_{h} \in U_{h}} \frac{b\left(p_{h}, u_{h}\right)}{\left\|u_{h}\right\|} \geq c_{b}\left\|p_{h}\right\|, \quad p_{h} \in P_{h} .
$$

2. There exists $C>0$ such that $\left\|u_{h}\right\|_{U / Z_{h}} \leq C\left\|u_{h}\right\|_{U / \hat{Z}_{h}}$ for all $u_{h} \in U_{h}$, and

$$
\sup _{u_{h} \in U_{h}} b\left(p_{h}, u_{h}\right)>0, \quad p_{h} \neq 0 .
$$

In either case there exists $C>0$ such that

$$
\inf _{z_{h} \in Z_{h}}\left\|u-z_{h}\right\| \leq C \inf _{v_{h} \in U_{h}}\left\|u-v_{h}\right\| \quad \text { for all } u \in \hat{Z}_{h} .
$$

As stated previously, the form of the error estimates for a specific problem depends upon the structure of the nonlinear operator $a(.,$.$) . The following theorem considers the simplest situation$ where the operator is (strictly) maximal monotone and Lipschitz continuous (in which case $U$ is typically a Hilbert space).

Theorem 2.2 Let $U$ and $P$ be reflexive Banach spaces, $b: P \times U \rightarrow \mathbb{R}$ be continuous and bilinear, and let $a: U \times U \rightarrow \mathbb{R}$ be linear in its second argument. Assume that $b(.,$.$) satisfies the inf-sup$ condition: there exists $c_{b}>0$ such that

$$
\sup _{v \in U} \frac{b(p, v)}{\|v\|_{U}} \geq c_{b}\|p\|_{P}
$$

Assume additionally that $a(.,$.$) is strictly monotone and Lipschitz in its first argument:$

$$
c\|u-v\|_{U}^{2} \leq a(u, u-v)-a(v, u-v), \quad \text { and } \quad a(u, w)-a(v, w) \leq C\|u-v\|_{U}\|w\|_{U} .
$$

Let $U_{h} \subset U$ and $P_{h} \subset P$ be subspaces and suppose that $b: U_{h} \times P_{h} \rightarrow \mathbb{R}$ satisfies the inf-sup condition: there exists $c_{b}>0$ (independent of $h$ ) such that

$$
\sup _{v_{h} \in U_{h}} \frac{b\left(p_{h}, v_{h}\right)}{\left\|v_{h}\right\|_{U}} \geq c_{b}\left\|p_{h}\right\|_{P}
$$


Fix $(f, g) \in U^{\prime} \times P^{\prime}$ and suppose that $(u, p) \in U \times P$ satisfies

$$
a(u, v)-b(p, v)=f(v), \quad b(q, u)=g(q), \quad(v, q) \in U \times P,
$$

and suppose $\left(u_{h}, p_{h}\right) \in U_{h} \times P_{h}$ satisfies

$$
a\left(u_{h}, v_{h}\right)-b\left(p_{h}, v_{h}\right)=f\left(v_{h}\right), \quad b\left(q_{h}, u_{h}\right)=g\left(q_{h}\right), \quad\left(v_{h}, q_{h}\right) \in U_{h} \times P_{h} .
$$

Then there exists a constant $C>0$ such that

$$
\left\|u-u_{h}\right\|_{U}+\left\|p-p_{h}\right\|_{P} \leq C\left\{\inf _{v_{h} \in U_{h}}\left\|u-v_{h}\right\|_{U}+\inf _{q_{h} \in P_{h}}\left\|p-q_{h}\right\|_{P}\right\}
$$

Proof Using the monotonicity and continuity properties of $a(.,$.$) and the Galerkin orthogonality$ condition (2.4) shows

$$
\begin{aligned}
c\left\|u-u_{h}\right\|_{U}^{2} & \leq a\left(u, u-u_{h}\right)-a\left(u_{h}, u-u_{h}\right) \\
& \leq a\left(u, u-v_{h}+v_{h}-u_{h}\right)-a\left(u_{h}, u-v_{h}+v_{h}-u_{h}\right) \\
& \leq C\left\|u-u_{h}\right\|_{U}\left\|u-v_{h}\right\|_{U}+b\left(p-p_{h}, v_{h}-u_{h}\right) .
\end{aligned}
$$

If $v_{h} \in u_{h}+Z_{h}$, then

$$
\begin{aligned}
c\left\|u-u_{h}\right\|_{U}^{2} & \leq C\left\|u-u_{h}\right\|_{U}\left\|u-v_{h}\right\|_{U}+b\left(p-q_{h}, v_{h}-u_{h}\right) \quad q_{h} \in P_{h} \\
& \leq C\left(\left\|u-v_{h}\right\|_{U}+\left\|p-q_{h}\right\|_{P}\right)^{2}+(c / 2)\left\|u-u_{h}\right\|_{U}^{2} .
\end{aligned}
$$

It follows that

$$
\left\|u-u_{h}\right\|_{U} \leq C\left(\left\|u-v_{h}\right\|_{U}+\left\|p-q_{h}\right\|_{P}\right), \quad v_{h} \in u_{h}+Z_{h}, q_{h} \in P_{h} .
$$

Adopting the notation of Lemma 2.2 we have $u-v_{h}=u-u_{h}-z_{h}$ for $z_{h} \in Z_{h}$ and $u-u_{h} \in \hat{Z}_{h}$, in which case the lemma can be used to obtain

$$
\left\|u-u_{h}\right\|_{U} \leq C \inf _{\left(v_{h}, q_{h}\right) \in U_{h} \times P_{h}}\left(\left\|u-v_{h}\right\|_{U}+\left\|p-q_{h}\right\|_{P}\right) .
$$

The second statement of Lemma 2.1 and the Lipschitz continuity assumption on $a(.,$.$) show$

$$
\begin{aligned}
\left\|p-p_{h}\right\|_{P} & \leq(1+C / c)\left\|p-q_{h}\right\|_{P}+(1 / c) \sup _{v_{h} \in U_{h}} \frac{a\left(u, v_{h}\right)-a\left(u_{h}, v_{h}\right)}{\left\|v_{h}\right\|_{U}} \\
& \leq(1+C / c)\left\|p-q_{h}\right\|_{P}+C\left\|u-u_{h}\right\|_{U} \\
& \leq C \inf _{\left(v_{h}, q_{h}\right) \in U_{h} \times P_{h}}\left(\left\|u-u_{h}\right\|_{U}+\left\|p-q_{h}\right\|_{P}\right) .
\end{aligned}
$$

\section{Twofold Saddle Point Problems}

In this section, the twofold saddle point problems (1.1) and (1.2) are considered, and equivalent conditions for the existence and uniqueness of solutions are formulated. 


\subsection{Problem Type 1}

In this section we consider the twofold saddle problem arising from (1.1): find $\left(u, p_{1}, p_{2}\right) \in U \times P_{1} \times P_{2}$ such that

$$
\begin{aligned}
a(u, v)-b_{1}\left(p_{1}, v\right)-b_{2}\left(p_{2}, v\right) & =f(v) & & \forall v \in U, \\
b_{1}\left(q_{1}, u\right) & & =g_{1}\left(q_{1}\right) & \forall q_{1} \in P_{1}, \\
b_{2}\left(q_{2}, u\right) & & =g_{2}\left(q_{2}\right) & \forall q_{2} \in P_{2} .
\end{aligned}
$$

This can be viewed as a single saddle point problem on $\left(P_{1} \times P_{2}\right) \times U$ with bilinear form

$$
b\left(\left(p_{1}, p_{2}\right), u\right)=b_{1}\left(p_{1}, u\right)+b_{2}\left(p_{2}, u\right) .
$$

The following lemma provides several criteria which guarantee that this bilinear form satisfies the inf-sup condition. Frequently one of these criteria is more readily verified for the discrete spaces used a particular numerical scheme. This lemma extends results in [28] and [20].

Lemma 3.1 Let $U, P_{1}$, and $P_{2}$ be reflexive Banach spaces, and let $b: P_{1} \times U \rightarrow \mathbb{R}$, and $b_{2}: P_{2} \times U \rightarrow$ $\mathbb{R}$ be bilinear and continuous. Let

$$
Z_{b_{i}}=\left\{v \in U \mid b_{i}\left(q_{i}, v\right)=0 \forall q_{i} \in P_{i}\right\} \subset U, \quad i=1,2,
$$

then the following are equivalent:

(1) There exists $c>0$ such that

$$
\sup _{v \in U} \frac{b_{1}\left(p_{1}, v\right)+b_{2}\left(p_{2}, v\right)}{\|v\|_{U}} \geq c\left(\left\|p_{1}\right\|_{P_{1}}+\left\|p_{2}\right\|_{P_{2}}\right) \quad\left(p_{1}, p_{2}\right) \in P_{1} \times P_{2}
$$

(2) There exists $c>0$ such that

$$
\sup _{v \in U} \frac{b_{1}\left(p_{1}, v\right)}{\|v\|_{U}} \geq c\left\|p_{1}\right\|_{P_{1}}, p_{1} \in P_{1} \quad \text { and } \quad \sup _{v \in Z_{b_{1}}} \frac{b_{2}\left(p_{2}, v\right)}{\|v\|_{U}} \geq c\left\|p_{2}\right\|_{P_{2}}, p_{2} \in P_{2}
$$

(3) There exists $c>0$ such that

$$
\sup _{v \in Z_{b_{2}}} \frac{b_{1}\left(p_{1}, v\right)}{\|v\|_{U}} \geq c\left\|p_{1}\right\|_{P_{1}}, p_{1} \in P_{1} \quad \text { and } \quad \sup _{v \in U} \frac{b_{2}\left(p_{2}, v\right)}{\|v\|_{U}} \geq c\left\|p_{2}\right\|_{P_{2}}, p_{2} \in P_{2}
$$

(4) There exists $c>0$ such that

$$
\sup _{v \in Z_{b_{2}}} \frac{b_{1}\left(p_{1}, v\right)}{\|v\|_{U}} \geq c\left\|p_{1}\right\|_{P_{1}}, p_{1} \in P_{1} \quad \text { and } \quad \sup _{v \in Z_{b_{1}}} \frac{b_{2}\left(p_{2}, v\right)}{\|v\|_{U}} \geq c\left\|p_{2}\right\|_{P_{2}}, p_{2} \in P_{2} .
$$

Proof The result is shown by proving $(4) \Rightarrow(2) \Rightarrow(1) \Rightarrow(4)$ and $(4) \Rightarrow(3) \Rightarrow(1) \Rightarrow(4)$. The implications $(4) \Rightarrow(2)$ and $(4) \Rightarrow(3)$ are clear.

As shown in [20], to prove $(2) \Rightarrow(1)$ note that (2) implies the existence of $v_{1} \in U$ with $\left\|v_{1}\right\|_{U}=1$ and $b_{1}\left(q_{1}, v_{1}\right) \geq(c / 2)\left\|q_{1}\right\|_{P_{1}}$, and the existence of $v_{2} \in Z_{b_{2}}$ with $\left\|v_{2}\right\|_{U}=1$ and $b_{2}\left(q_{2}, v_{2}\right) \geq$ $(c / 2)\left\|q_{2}\right\|_{P_{2}}$. Since $b_{2}(\cdot, \cdot)$ is continuous, there is a $C>0$ such that $b_{2}\left(q_{2}, v\right) \leq C\left\|q_{2}\right\|_{P_{2}}\|v\|_{U}$ for all $\left(q_{2}, v\right) \in P_{2} \times U$. Set $u=v_{1}+(1+2 C / c) v_{2}$. Then $\|u\|_{U} \leq 2(1+2 C / c)$,

$$
b_{1}\left(q_{1}, u\right)=b_{1}\left(q_{1}, v_{1}\right) \geq \frac{c}{2}\left\|q_{1}\right\|_{P_{1}}
$$


and

$$
\begin{aligned}
b_{2}\left(q_{2}, u\right) & =b_{2}\left(q_{2}, v_{1}\right)+\left(1+\frac{2 C}{c}\right) b_{2}\left(q_{2}, v_{2}\right) \\
& \geq-C\left\|q_{2}\right\|_{P_{2}}+\left(1+\frac{2 C}{c}\right) \frac{c}{2}\left\|q_{2}\right\|_{P_{2}} \\
& =\frac{c}{2}\left\|q_{2}\right\|_{P_{2}} .
\end{aligned}
$$

Then

$$
\frac{b_{1}\left(q_{1}, u\right)+b_{2}\left(q_{2}, u\right)}{\|u\|_{U}} \geq \frac{c}{4(1+2 C / c)}\left(\left\|q_{1}\right\|_{P_{1}}+\left\|q_{2}\right\|_{P_{2}}\right)
$$

proving (1). The proof that $(3) \Rightarrow(1)$ is similar.

To complete the equivalence, assume (1) and let

$$
Z=Z_{b_{1}} \cap Z_{b_{2}}=\left\{u \in U \mid b_{1}\left(p_{1}, u\right)+b_{2}\left(p_{2}, u\right)=0,\left(p_{1}, p_{2}\right) \in P_{1} \times P_{2}\right\} .
$$

The generalized Lax Milgram theorem then shows that for each $f \in(U / Z)^{\prime}$ there exists a unique $\left(p_{1}, p_{2}\right) \in P_{1} \times P_{2}$ satisfying

$$
b_{1}\left(p_{1}, v\right)+b_{2}\left(p_{2}, v\right)=f(v), \quad v \in U,
$$

with $\left(\left\|p_{1}\right\|_{P_{1}}+\left\|p_{2}\right\|_{P_{2}}\right) \leq(1 / c)\|f\|_{U^{\prime}}$. Here we identify $(U / Z)^{\prime} \simeq\left\{f \in U^{\prime} \mid f(u)=0, u \in Z\right\}$.

It follows that for each $f \in(U / Z)^{\prime}$ there exists $p_{2} \in P_{2}$ satisfying $b_{2}\left(p_{2}, v\right)=f(v)$ for all $v \in Z_{b_{1}}$ with $\left\|p_{2}\right\|_{P_{2}} \leq(1 / c)\|f\|_{U^{\prime}}$. The Lax-Milgram condition then shows that $b_{2}$ is coercive on $P_{2} \times Z_{b_{1}} / Z$ so satisfies the inf-sup condition stated in (4).

Similarly for each $f \in(U / Z)^{\prime}$ there exists $p_{1} \in P_{1}$ satisfying $b_{1}\left(p_{1}, v\right)=f(v)$ for all $v \in Z_{b_{2}}$ with $\left\|p_{1}\right\|_{P_{1}} \leq(1 / c)\|f\|_{U^{\prime}}$ so $b_{1}$ satisfies the inf-sup condition stated in (4).

Combining Lemma 3.1 and Theorem 2.1 gives necessary and sufficient conditions for twofold saddle problem (3.1).

Theorem 3.1 Let $U, P_{1}, P_{2}, b_{1}, b_{2}, Z_{b_{1}}$ and $Z_{b_{2}}$ be as in Lemma 3.1, and let $Z=Z_{b_{1}} \cap Z_{b_{2}}$. Assume $a: U \times U \rightarrow \mathbb{R}$ is linear in its second argument. Assume that for all $f \in U^{\prime}$ and $u_{g} \in U$ there exists $u_{0} \in Z$ such that

$$
a\left(u_{g}+u_{0}, v\right)=f(v) \quad v \in Z,
$$

and that one of the conditions (1)-(4) of Lemma 3.1 are satisfied. Then for all $f \in U^{\prime}, g_{1} \in P_{1}^{\prime}$, and $g_{2} \in P_{2}^{\prime}$ there exists $\left(u, p_{1}, p_{2}\right) \in U \times P_{1} \times P_{2}$ satisfying (3.1) with $\|u\|_{U / Z} \leq C\left(\left\|g_{1}\right\|_{P_{1}^{\prime}}+\left\|g_{2}\right\|_{P_{2}^{\prime}}\right)$.

Remark 3.1 Problems of the form

$$
a(u, v)-\sum_{j=1}^{k} b_{j}\left(p_{j}, v\right)=f(v), \quad \sum_{i=1}^{k} b_{i}\left(q_{i}, u\right)=\sum_{i=1}^{k} g_{i}\left(q_{i}\right)
$$

for all $\left(v, q_{1}, \ldots, q_{k}\right) \in U \times P_{1} \times \cdots \times P_{k}$ are considered in [20] and [28]. 


\subsection{Problem Type 2}

We next consider the twofold saddle problems (1.2) and (1.3) corresponding to the variational problem $\left(u, p_{1}, p_{2}\right) \in U \times P_{1} \times P_{2}$,

$$
\begin{aligned}
a(u, v)-b_{1}\left(p_{2}, v\right) & =f(v) & & \forall v \in U, \\
-b_{2}\left(p_{2}, q_{1}\right) & =g_{1}\left(q_{1}\right) & & \forall q_{1} \in P_{1}, \\
b_{1}\left(q_{2}, u\right)+b_{2}\left(q_{2}, p_{1}\right) & =g_{2}\left(q_{2}\right) & & \forall q_{2} \in P_{2} .
\end{aligned}
$$

As indicated in the introduction, two different single saddle problems result with different groupings of the variables. This gives rise to different, but equivalent, statements of the inf-sup conditions required for the existence of solutions. Different characterizations of the inf-sup condition are useful when constructing numerical schemes since one form is frequently easier to verify than the other.

Define

$$
Z_{b_{2}}=\left\{q_{2} \in P_{2} \mid b_{2}\left(q_{2}, p_{1}\right)=0, p_{1} \in P_{1}\right\} \quad \text { and } \quad \mathcal{D}=\left\{u \in U \mid b_{1}\left(q_{2}, u\right)=0, q_{2} \in Z_{b_{2}}\right\} .
$$

Grouping the variables as $\left(U \times P_{1}\right) \times P_{2}$ gives rise to a single saddle problem with operator having block form $\left[\begin{array}{cc}A & 0 \\ 0 & 0\end{array}\right]$. Coercivity of this operator on $Z \subset U \times P_{1}$ will require $\left\|p_{1}\right\|_{P_{1}} \leq C\|u\|_{U}$ when $\left(u, p_{1}\right) \in Z$. Alternately stated, $Z$ is the graph of the operator $B_{2}^{-1} B_{1}: \mathcal{D} \subset U \rightarrow P_{1}$, and the inf-sup condition on $b_{2}$ guarantees that this operator is well-defined and continuous. This is the content of statement (1) of Lemma 3.2 below.

With the grouping $\left(U \times P_{2}\right) \times P_{1}$ problem (3.2) has the form of the single saddle problem, the solution of which requires $b_{2}(\cdot, \cdot)$ to satisfy an inf-sup condition on $P_{2}$ and the operator defined by the block $\left[\begin{array}{cc}A & -B_{1}^{T} \\ B_{1} & 0\end{array}\right]$ must be coercive over $U \times Z_{b_{2}}$. This latter condition requires $b_{1}(.,$.$) to satisfy an$ inf-sup condition on $Z_{b_{2}}$. These two inf-sup conditions correspond to statement (2) of the following lemma.

The inf-sup conditions in statement (3) of the lemma arose in one approach to proving an error estimate for the twofold saddle problem [19]. The first condition gives the existence of a projection operator necessary to ensure that the best approximation of $\left(u, p_{1}\right)$ can be lifted from the discrete kernel $Z_{h}$ to $U_{h} \times P_{1 h}$, while the second condition provides the same lifting for approximations of $p_{2}$ from $Z_{b_{2} h}$ to $P_{2 h}$.

Lemma 3.2 Let $U, P_{1}, P_{2}$ be reflexive Banach spaces, and $b_{1}: P_{2} \times U \rightarrow \mathbb{R}$ and $b_{2}: P_{2} \times P_{1} \rightarrow \mathbb{R}$ be bilinear and continuous. Define the bilinear form $b: P_{2} \times\left(U \times P_{1} \rightarrow \mathbb{R}\right.$ by $b\left(p_{2},\left(u, p_{1}\right)\right)=$ $b_{1}\left(p_{2}, u\right)+b_{2}\left(p_{2}, p_{1}\right)$, and let

$$
Z_{b_{2}}=\left\{q_{2} \in P_{2} \mid b_{2}\left(q_{2}, p_{1}\right)=0, p_{1} \in P_{1}\right\},
$$

and

$$
Z_{b}=\left\{\left(u, p_{1}\right) \in U \times P_{1} \mid b\left(p_{2},\left(u, p_{1}\right)\right)=0, p_{2} \in P_{2}\right\} .
$$

Then the following are equivalent:

(1) There exists a constants $C$ and $c>0$ such that

$$
\sup _{\left(v, q_{1}\right) \in U \times P_{1}} \frac{b\left(p_{2},\left(v, q_{1}\right)\right)}{\left\|\left(v, q_{1}\right)\right\|_{U \times P_{1}}} \geq c\left\|p_{2}\right\|_{P_{2}} \quad \text { and } \quad\left\|q_{1}\right\|_{P_{1}} \leq C\|v\|_{U} \text { for }\left(v, q_{1}\right) \in Z_{b} .
$$

(2) There exists $c>0$ such that

$$
\sup _{p_{2} \in P_{2}} \frac{b_{2}\left(p_{2}, q_{1}\right)}{\left\|p_{2}\right\|_{P_{2}}} \geq c\left\|q_{1}\right\|_{P_{1}}, \quad \text { and } \quad \sup _{v \in U} \frac{b_{1}\left(p_{2}, v\right)}{\|v\|_{U}} \geq c\left\|p_{2}\right\|_{P_{2}}, \quad \text { for } p_{2} \in Z_{b_{2}} \text {. }
$$


(3) There exists $c>0$ such that

$$
\sup _{\left(v, q_{1}\right) \in U \times P_{1}} \frac{b\left(p_{2},\left(v, q_{1}\right)\right)}{\left\|\left(v, q_{1}\right)\right\|_{U \times P_{1}}} \geq c\left\|p_{2}\right\|_{P_{2}}, \quad \text { and } \quad \sup _{p_{2} \in P_{2}} \frac{b_{2}\left(p_{2}, q_{1}\right)}{\left\|p_{2}\right\|_{P_{2}}} \geq c\left\|q_{1}\right\|_{P_{1}} .
$$

Moreover, when one of these conditions holds $Z_{b}$ is the graph of a linear function with domain

$$
\mathcal{D}=\left\{u \in U \mid b_{1}\left(q_{2}, u\right)=0, q_{2} \in Z_{b_{2}}\right\}=\left\{u \mid\left(u, p_{1}\right) \in Z_{b}\right\} .
$$

Remark: The inclusion $\left\{u \mid\left(u, p_{1}\right) \in Z_{b}\right\} \subset\left\{u \in U \mid b_{1}\left(q_{2}, u\right)=0, q_{2} \in Z_{b_{2}}\right\}$ always holds; the reverse inclusion requires $b_{2}$ to satisfy an inf-sup condition.

Proof We show $(1) \Rightarrow(2) \Rightarrow(3) \Rightarrow(1)$.

$(1) \Rightarrow(2)$ Setting $p_{2} \in Z_{b_{2}}$ in the inf-sup condition satisfied by $b(.,$.$) immediately gives the$ inf-sup condition on $b_{1}(.,$.$) sated in (2). To establish the inf-sup condition on b_{2}(.,$.$) use adjoint$ coercivity of $b: P_{2} \times\left(U \times P_{1}\right) / Z_{b}$ to obtain

$$
\sup _{p_{2} \in P_{2}} \frac{b\left(p_{2},\left(u, p_{1}\right)\right)}{\left\|p_{2}\right\|_{P_{2}}} \geq c\left\|\left(u, p_{1}\right)\right\|_{\left(U \times P_{1}\right) / Z_{b}} .
$$

Setting $u=0$ and expanding the definition of the quotient norm shows

$$
\begin{aligned}
\sup _{p_{2} \in P_{2}} \frac{b_{2}\left(p_{2}, p_{1}\right)}{\left\|p_{2}\right\|_{P_{2}}} & \geq c \inf _{\left(v, q_{1}\right) \in Z_{b}}\left(\|v\|_{U}+\left\|p_{1}-q_{1}\right\|_{P_{1}}\right) \\
& \geq(c / C) \inf _{\left(v, q_{1}\right) \in Z}\left(\left\|q_{1}\right\|_{P_{1}}+\left\|p_{1}-q_{1}\right\|_{P_{1}}\right) \\
& \geq(c / C)\left\|p_{1}\right\|_{P_{1}} .
\end{aligned}
$$

The second line follows from the property assumed upon elements of $Z_{b}$ and it was assumed without loss of generality that $C \geq 1$.

$(2) \Rightarrow(3)$ We first show that for each $g_{2} \in P_{2}^{\prime}$ there exists a solution of $\left(v, q_{1}\right) \in U \times P_{1}$ of

$$
b\left(q_{2},\left(v, q_{1}\right)\right) \equiv b_{1}\left(q_{2}, v\right)+b_{2}\left(q_{2}, q_{1}\right)=g_{2}\left(q_{2}\right), \quad q_{2} \in P_{2},
$$

with $c\left\|\left(v, q_{1}\right)\right\|_{U \times P_{1}} \leq\left\|g_{2}\right\|_{P_{2}^{\prime}}$. The inf-sup assumed on $b_{1}(.,$.$) shows that its restriction to b_{1}$ : $Z_{b_{2}} \times U / \mathcal{D} \rightarrow \mathbb{R}$ is coercive where

$$
\mathcal{D}=\left\{u \in U \mid b_{1}\left(q_{2}, u\right)=0, q_{2} \in Z_{b_{2}}\right\} .
$$

It follows that there exists $v \in U$ such that

$$
b_{1}\left(q_{2}, v\right)=g_{2}\left(q_{2}\right) \quad q_{2} \in Z_{b_{2}}
$$

with $c\|v\|_{U} \leq\left\|g_{2}\right\|_{P_{2}^{\prime}}$. Next, the inf-sup condition on $b_{2}(\cdot, \cdot)$ shows that $b_{2}: P_{2} / Z_{b_{2}} \times P_{1} \rightarrow \mathbb{R}$ is coercive. Since $g_{2}\left(q_{2}\right)-b_{1}\left(q_{2}, v\right)$ vanishes for $q_{2} \in Z_{b_{2}}$ there exists a solution $q_{1}$ of

$$
b_{2}\left(q_{2}, q_{1}\right)=g_{2}\left(q_{2}\right)-b_{1}\left(q_{2}, v\right) \quad q_{2} \in P_{2},
$$

with

$$
c\left\|q_{1}\right\|_{P_{1}} \leq\left\|g_{2}\right\|+C\|v\|_{U} \leq(1+C / c)\left\|g_{2}\right\|_{P_{2}^{\prime}} .
$$

This gives a solution of equation (3.3) with $c\left\|\left(v, q_{1}\right)\right\|_{U \times P_{1}} \leq\left\|g_{2}\right\|_{P_{2}^{\prime}}$. 
To establish the inf-sup condition on $b$ in (3), fix $p_{2} \in P_{2}$ and let $g_{2} \in P_{2}^{\prime}$ satisfy $g_{2}\left(p_{2}\right)=\left\|g_{1}\right\|_{P^{\prime}}^{2}=$ $\left\|p_{2}\right\|_{P_{2}}^{2}$. Then the solution of (3.3) satisfies $c\left\|\left(v, q_{1}\right)\right\|_{U \times P_{1}} \leq\left\|g_{2}\right\|_{P_{2}^{\prime}}=\left\|p_{2}\right\|_{P_{2}}$ and

$$
\left\|p_{2}\right\|_{P_{2}}=\frac{g_{2}\left(p_{2}\right)}{\left\|p_{2}\right\|_{P_{2}}}=\frac{b\left(p_{2},\left(v, q_{1}\right)\right)}{\left\|p_{2}\right\|_{P_{2}}} \leq \frac{b\left(p_{2},\left(v, q_{1}\right)\right)}{c\left\|\left(v, q_{1}\right)\right\|_{U \times P_{1}}} .
$$

$(3) \Rightarrow(1)$ Adjoint coercivity of $b: P_{2} \times\left(U \times P_{1}\right) \rightarrow \mathbb{R}$ shows

$$
\sup _{p_{2} \in P_{2}} \frac{b\left(p_{2},\left(v, q_{1}\right)\right)}{\left\|p_{2}\right\|_{P_{2}}} \geq c\left\|\left(v, q_{1}\right)\right\|_{\left(U \times P_{1}\right) / Z_{b}} .
$$

If $\left(v, q_{1}\right) \in Z_{b}$ it follows that $b\left(p_{2},\left(v, q_{1}\right)\right)=0$ for all $p_{2} \in P_{2}$; that is, $b_{2}\left(p_{2}, q_{1}\right)=-b_{1}\left(p_{2}, v\right)$ for all $p_{2} \in P_{2}$. The coercivity assumed on $b_{2}(.,$.$) then shows$

$$
c\left\|q_{1}\right\|_{P_{1}} \leq \sup _{p_{2} \in P_{2}} \frac{b_{2}\left(p_{2}, q_{1}\right)}{\left\|p_{2}\right\|_{P_{2}}}=\sup _{p_{2} \in P_{2}} \frac{-b_{1}\left(p_{2}, v\right)}{\left\|p_{2}\right\|_{P_{2}}} \leq C\|v\|_{U} .
$$

It follows that problems of the form (3.2) can be treated as single saddle point problems and the application of Theorem 2.1 gives rise to necessary and sufficient conditions for existence of solutions.

Theorem 3.2 Let $U, P_{1}, P_{2}, b_{1}, b_{2}, \mathcal{D}$ be as in Lemma 3.2 and assume $a: U \times U \rightarrow \mathbb{R}$ is linear in its second argument. Assume that for all $f \in U^{\prime}$ and $u_{g} \in U$ there exists $u_{0} \in D$ such that

$$
a\left(u_{g}+u_{0}, v\right)=f(v) \quad v \in D,
$$

and that one of the conditions (1)-(3) of Lemma 3.2 are satisfied. Then for all $f \in U^{\prime}, g_{1} \in P_{1}^{\prime}$, and $g_{2} \in P_{2}^{\prime}$ there exists $\left(u, p_{1}, p_{2}\right) \in U \times P_{1} \times P_{2}$ satisfying (3.2) with $\|u\|_{U / \mathcal{D}} \leq C\left(\left\|g_{1}\right\|_{P_{1}^{\prime}}+\left\|g_{2}\right\|_{P_{2}^{\prime}}\right)$.

\section{Application to Problem (1.5)}

In this section the saddle point theory developed above is used to solve the nonlinear Stokes problem (1.5) and to formulate numerical schemes to approximate the solution.

Assume $\Omega \subset \mathbb{R}^{d}, d=2,3$, is bounded and simply connected and $\partial \Omega=\Gamma_{0} \cup \Gamma_{1}$ is Lipschitzcontinuous and the boundary partition is sufficiently regular for classical $H^{2}(\Omega)$ regularity to apply. Let

$$
\begin{gathered}
\mathbb{S}=\left\{S \in \mathbb{H}(\Omega ; \operatorname{div})_{\text {sym }}|S \mathbf{n}|_{\Gamma_{1}}=0\right\}, \quad U=L^{2}(\Omega)^{d}, \\
\mathbb{D}=\left\{D \in L^{2}(\Omega)_{\text {sym }}^{d \times d} \mid \operatorname{tr}(D)=0\right\} .
\end{gathered}
$$

For simplicity we assume a homogeneous traction boundary condition $\Gamma_{1}$; non-homogeneous boundary data can be accommodated with the usual translation argument. The variational problem is: given $f \in L^{2}(\Omega)^{d}$ and $\mathbf{u}_{0} \in H^{1 / 2}\left(\Gamma_{0}\right)^{d}$, find $(D, \mathbf{u}, S) \in \mathbb{D} \times U \times \mathbb{S}$ satisfying

$$
\begin{aligned}
\int_{\Omega} \mathcal{A}(D): E-S: E & =0, \quad E \in \mathbb{D} \\
\int_{\Omega}-\operatorname{div}(S) \cdot \mathbf{v} & =\int_{\Omega} f \cdot \mathbf{v} . \quad \mathbf{v} \in U . \\
\int_{\Omega}(D: T+\mathbf{u} \cdot \operatorname{div}(T)) & =\int_{\Gamma_{0}} \mathbf{u}_{0} \cdot T \mathbf{n}, \quad T \in \mathbb{S},
\end{aligned}
$$

This problem takes the form of the twofold saddle problem considered in Section 3.2 with operators

$$
a(D, E)=\int_{\Omega} \mathcal{A}(D): E, \quad b_{1}(S, E)=\int_{\Omega} S: E, \quad b_{2}(S, \mathbf{v})=\int_{\Omega} \operatorname{div}(S) . \mathbf{v} .
$$

Below we will assume $\left|\Gamma_{1}\right| \neq 0$, otherwise it is necessary to require $S \in \mathbb{S}$ to satisfy $\int_{\Omega} \operatorname{tr}(S)=0$. 


\subsection{Solution of the Continuous Problem}

Theorem 3.2 will be used to solve problem (4.1) using condition (2) of Lemma 3.2 to establish the inf-sup hypotheses.

First Inf-Sup Condition: The inf-sup condition on $b_{2}(.,$.$) becomes$

$$
\sup _{S \in \mathbb{S}} \frac{\int_{\Omega} \operatorname{div}(S) \cdot \mathbf{v}}{\|S\|_{H(\Omega ; \operatorname{div})}} \geq c\|\mathbf{v}\|_{L^{2}(\Omega)}, \quad \forall \mathbf{v} \in U
$$

A proof of this inequality may be found in $[12, \S 11.1,11.2]$. Briefly, given $\mathbf{v} \in U$ select $S=D(\mathbf{u}) \equiv$ $(1 / 2)\left(\nabla \mathbf{u}+(\mathbf{u})^{T}\right)$ where $\mathbf{u}$ is the solution of the linear elasticity problem,

$$
\operatorname{div}(D(\mathbf{u}))=\mathbf{v},\left.\quad \mathbf{u}\right|_{\Gamma_{0}}=0,\left.\quad D(\mathbf{u}) \mathbf{n}\right|_{\Gamma_{1}}=\mathbf{0} .
$$

Then $\int_{\Omega} \operatorname{div}(S) \cdot \mathbf{v}=\|\mathbf{v}\|_{L^{2}(\Omega)}^{2}$ the Poincaré and Korn inequalities may be used to verify that there exists $C>0$ such that $\|S\|_{H(\Omega \text {; div })} \leq C\|\mathbf{v}\|_{L^{2}(\Omega)}$, and (4.2) follows. For the discrete problem we use the property that regularity theory for the linear elastic problem shows that if $\mathbf{v} \in L^{2}(\Omega)^{d}$ then $\mathbf{u} \in H^{2}(\Omega)^{d}$ so $S=D(\mathbf{u}) \in H^{1}(\Omega)^{d \times d}$.

Second Inf-Sup Condition: Setting $Z_{b_{2}}=\{S \in \mathbb{S} \mid \operatorname{div} S=0\}$, the inf-sup condition required of $b_{1}(.,$.$) becomes$

$$
\sup _{D \in \mathbb{D}} \frac{\int_{\Omega} D: S}{\|D\|_{L^{2}(\Omega)}} \geq c\|S\|_{L^{2}(\Omega)}, \quad \forall S \in Z_{b_{2}} .
$$

Given $S \in Z_{b_{2}}$ the inf-sup condition is established upon setting $D=S-(1 / e) \operatorname{tr}(S) \in \mathbb{D}$ to obtain $\int_{\Omega} D: S=\|D\|_{L^{2}(\Omega)}^{2}$. Lemma A.2 in the appendix shows that there is a constant $C>0$ such that $\|S\|_{L^{2}(\Omega)} \leq C\|D\|_{L^{2}(\Omega)}$ when $S \in Z_{b_{2}}$ and (4.3) follows.

Define the set

$$
\mathcal{D}=\left\{D \in \mathbb{D} \mid \int_{\Omega} D: S=0 \quad \forall S \in Z_{b_{2}}\right\} \subset \mathbb{D} .
$$

This set is not easily characterized so minimal conditions on $\mathcal{A}: \mathbb{R}^{d \times d} \rightarrow \mathbb{R}^{d \times d}$ to guarantee coercivity over $\mathcal{D}$ are not available. Fortunately $\mathcal{A}$ is typically a maximal monotone operator on all of $\mathbb{R}^{d \times d}$ so this is not an issue. Granted this, Theorem 3.2 establishes existence of solutions to problem (4.1).

Theorem 4.1 Let $\Omega \subset \mathbb{R}^{d}$ and the spaces $\mathbb{S}, U$, and $\mathbb{D}$ be given at the beginning of this section. Assume $\mathcal{A}: \mathbb{R}^{d \times d} \rightarrow \mathbb{R}^{d \times d}$ maps bounded sets to bounded sets, and satisfies

$$
(\mathcal{A}(E)-\mathcal{A}(D)):(E-D) \geq 0 \quad \text { and } \quad \lim _{|D| \rightarrow \infty} \mathcal{A}(D): D /|D| \rightarrow \infty .
$$

Then for all $f \in L^{2}(\Omega)^{d}$ and $\mathbf{u}_{0} \in H^{1 / 2}\left(\Gamma_{0}\right)^{d}$, problem (4.1) has a solution $(D, \mathbf{u}, S) \in \mathbb{D} \times U \times \mathbb{S}$ satisfying

$$
\|D\|_{\mathbb{D} / \mathcal{D}} \leq C\left(\|\mathbf{f}\|_{L^{2}(\Omega)}+\left\|\mathbf{u}_{0}\right\|_{H^{1 / 2}\left(\Gamma_{0}\right)}\right)
$$

where

$$
\mathcal{D}=\left\{D \in \mathbb{D} \mid \int_{\Omega} D: S=0, \forall S \in \mathbb{S} \text { with } \operatorname{div}(S)=0\right\} .
$$

Moreover, if $\mathcal{A}$ is strictly monotone,

$$
(\mathcal{A}(E)-\mathcal{A}(D)):(E-D)>0, \quad E \neq D,
$$

the solution is unique. 


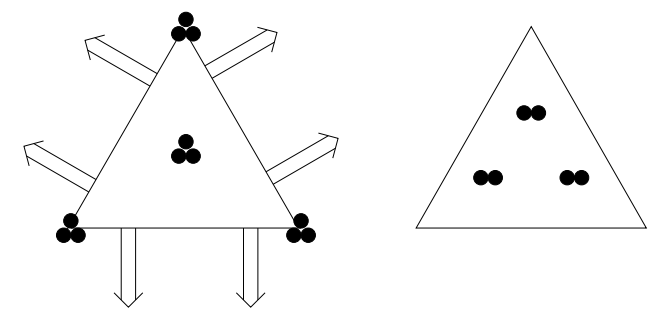

Fig. 4.1. Lowest-order Arnold-Winther elements in two dimensions for $S_{h}$ (left) and $u_{h}$ (right). For $S_{h}$, the points represent values of the components of $S_{h}$ (vertices) and the value of the three components of the moment of degree 0 of $S_{h}$ (interior), and the arrows represent the values of the moments of degree 0 and 1 of the two normal components of $S_{h}$ on each edge. For $u_{h}$, the points represent the value of of the two components at the three interior nodes.

\subsection{Galerkin Approximation}

Let $\left\{\mathcal{T}_{h}\right\}_{h>0}$ be a regular family of triangulations of $\Omega$ and let $\mathbb{D}_{h}, U_{h}, \mathbb{S}_{h}$ be finite dimensional subspaces of $\mathbb{D}, U, \mathbb{S}$, respectively. We consider Galerkin approximations of problem (4.1) satisfying $\left(D_{h}, \mathbf{u}_{h}, S_{h}\right) \in D_{h} \times U_{h} \times \mathbb{S}_{h}$,

$$
\begin{aligned}
\int_{\Omega} \mathcal{A}\left(D_{h}\right): E_{h}-S_{h}: E_{h} & =0, \quad E_{h} \in \mathbb{D}_{h} \\
\int_{\Omega}-\operatorname{div}\left(S_{h}\right) \cdot \mathbf{v}_{h} & =\int_{\Omega} f \cdot \mathbf{v}_{h}, \quad \mathbf{v}_{h} \in U_{h} \\
\int_{\Omega}\left(D_{h}: T_{h}+\mathbf{u}_{h} \cdot \operatorname{div}\left(T_{h}\right)\right) & =\int_{\Gamma_{0}} \mathbf{u}_{0} \cdot T_{h} \mathbf{n}, \quad T_{h} \in \mathbb{S}_{h},
\end{aligned}
$$

In this context establishing discrete versions of (4.2) and (4.3) can be considerably less complicated and technical than showing a condition of the form

$$
\sup _{\left(D_{h}, \mathbf{u}_{h}\right) \in \mathbb{D}_{h} \times U_{h}} \frac{\int_{\Omega}\left(D_{h}: T_{h}+\mathbf{u}_{h} \cdot \operatorname{div}\left(T_{h}\right)\right)}{\left\|D_{h}\right\|_{\mathbb{D}}+\left\|u_{h}\right\|_{U}} \geq c\left\|T_{h}\right\|_{H(\Omega ; \operatorname{div})},
$$

since the discrete form of (4.3) only needs to be verified for divergence free $T_{h}$.

First Inf-Sup Condition: The issue of finding conforming finite elements for symmetric tensors satisfying an inf-sup condition of the form (4.2) is well-documented $([14,32,4,5])$. We consider the finite element pairs $\left(\mathbb{S}_{h}, U_{h}\right)$ of symmetric tensors and vectors constructed by Arnold and Winther $[7,1]$ which satisfy the inf-sup condition.

Let $k \geq 1$ and define $\mathbb{P}_{k}(K)$ to be the set of all polynomials of degree at most $k$ on the simplex $K \in \mathcal{T}_{h}$. On $K$, define $\mathbb{S}_{K}$ to be the symmetric Arnold-Winther tensors

$$
\mathbb{S}_{K}=\left\{S_{h} \in \mathbb{H}(\operatorname{div}, K)_{\text {sym }} \mid S_{h} \in\left(\mathbb{P}_{k+d}(K)\right)_{\text {sym }}^{d \times d} \text { and } \operatorname{div}\left(S_{h}\right) \in\left(\mathbb{P}_{k}(K)\right\} .\right.
$$

The space $\mathbb{S}_{h}$ is the union of $\mathbb{S}_{K}$ over all $K \in \mathcal{T}_{h}$, subject to the condition that the normal components are continuous across mesh edges (faces for $d=3$ ) and all components are continuous at vertices. Define

$$
U_{h}=\left\{\mathbf{u} \in L^{2}(\Omega)^{d}|\mathbf{u}|_{K} \in \mathbb{P}_{k}(K) \quad \forall K \in \mathcal{T}_{h}\right\}
$$

and note that there is no interelement continuity requirement for $U_{h}$. Figure 4.1 gives a diagram of the degrees of freedom on each triangle for the lowest order Arnold-Winther $\left(S_{h}, u_{h}\right)$ pair $(k=1)$ in two dimensions. In $[7,1]$ an interpolation operator $\Pi_{h}: \mathbb{S} \cap H^{1}(\Omega)^{d \times d} \rightarrow \mathbb{S}_{h}$ is constructed for which 


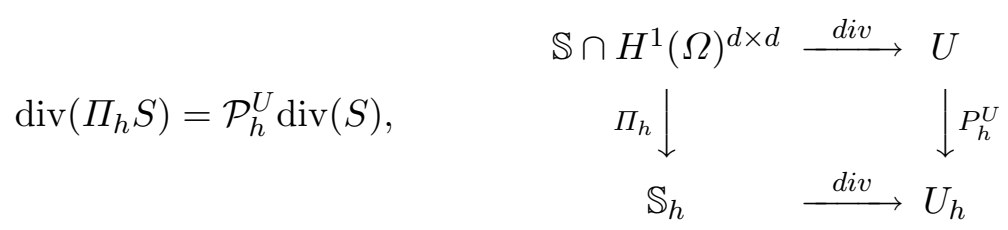

where is the orthogonal projection $\mathcal{P}_{h}^{U}: U \rightarrow U_{h}$ and $s>0$.

The discrete analog of (4.2) follows since for any $\mathbf{v}_{h} \in U_{h}$ there is a $S \in \mathbb{S} \cap H^{1}(\Omega)^{d \times d}$ satisfying $\operatorname{div}(S)=\mathbf{v}_{h}$ and $\|S\|_{L^{2}(\Omega)} \leq C\left\|\mathbf{v}_{h}\right\|_{L^{2}(\Omega)}$. Then $\operatorname{div}\left(\Pi_{h} S\right)=\mathcal{P}_{h}^{U} \operatorname{div}(S)=\mathbf{v}_{h}$ and $\left\|\Pi_{h} S\right\|_{H(\Omega ; \operatorname{div})} \leq$ $C\left\|\mathbf{v}_{h}\right\|_{U}$ and the discrete inf-sup condition follows.

Second Inf-Sup Condition: The commuting diagram for the Arnold Winther spaces shows

$$
Z_{h} \equiv\left\{S_{h} \in \mathbb{S}_{h} \mid \int_{\Omega} \operatorname{div}\left(S_{h}\right) \cdot \mathbf{u}_{h}=0, \quad \mathbf{u}_{h} \in U_{h}\right\}=\left\{S_{h} \in \mathbb{S}_{h} \mid \operatorname{div}\left(S_{h}\right)=\mathbf{0}\right\}
$$

The second inf-sup condition for the discrete spaces becomes

$$
\sup _{D_{h} \in \mathbb{D}_{h}} \frac{\int_{\Omega} D_{h}: S_{h}}{\left\|D_{h}\right\|_{L^{2}(\Omega)}} \geq c\left\|S_{h}\right\|_{L^{2}(\Omega)}, \quad S_{h} \in Z_{h} .
$$

When $\operatorname{div}(S)=0$, Lemma A.2 of the appendix shows that $\|S\|_{L^{2}(\Omega)} \leq C\left\|S_{0}\right\|_{L^{2}(\Omega)}$ where $S_{0}=$ $S-(\operatorname{tr}(S) / d) I$ is the trace free part of $S$. Since $S_{h} \in \mathbb{S}_{h}$ is piecewise polynomial of degree $k+d$ on $\mathcal{T}_{h}$, so it suffices to let

$$
\mathbb{D}_{h}=\left\{D_{h} \in L^{2}(\Omega)^{d \times d} \mid \operatorname{tr}\left(D_{h}\right)=0 \text { and }\left.D_{h}\right|_{K} \in \mathcal{P}_{k+d}(K)^{d \times d}, K \in \mathcal{T}_{h}\right\} ;
$$

however, typically much smaller spaces (and hence cheaper numerical schemes) suffice. Note too that smaller spaces will not necessarily result in a loss of accuracy. For smooth functions the Arnold Winther spaces exhibit the following approximation properties,

$$
\begin{aligned}
\left\|S-\Pi_{h} S\right\|_{\mathbb{H}(d i v ; \Omega)} & \leq C h^{k+1} \\
\left\|\mathbf{u}-\mathcal{P}_{h}^{U} \mathbf{u}\right\|_{L^{2}(\Omega)} & \leq C h^{k+1},
\end{aligned}
$$

and this rate would be achieved for $\left\|D_{h}-D\right\|_{L^{2}(\Omega)}$ if $\mathbb{D}_{h}$ contained the piecewise polynomials of degree $k$.

Having constructed discrete subspaces which satisfy these inf-sup conditions, Theorem 2.2 provides error estimates for the Galerkin approximations.

Theorem 4.2 Let $\Omega \subset \mathbb{R}^{d}$ and the spaces $\mathbb{S}, U$, and $\mathbb{D}$ be given at the beginning of this section. Assume there exist constants $C, c>0$ such that $\mathcal{A}: \mathbb{R}^{d \times d} \rightarrow \mathbb{R}^{d \times d}$ satisfies

$$
c|D-E|^{2} \leq(\mathcal{A}(E)-\mathcal{A}(D)):(E-D) \quad \text { and } \quad(\mathcal{A}(E)-\mathcal{A}(D)): F \leq|E-D||F| .
$$

Let $\left\{\mathcal{T}_{h}\right\}_{h>0}$ be a regular family of triangulations of $\Omega, \mathbb{S}_{h} \subset \mathbb{S}$ be the Arnold Winther space of index $k$ over $\mathcal{T}_{h}, U_{h} \subset U$ be the discontinuous piecewise polynomial space of degree $k$, and $\mathbb{D}_{h} \subset \mathbb{D}$ be the discontinuous piecewise polynomial space of degree $k+d$.

Let $(D, \mathbf{u}, S)$ satisfy (4.1) and $\left(D_{h}, \mathbf{u}_{h}, S_{h}\right)$ satisfy (4.4). Then there is a constant $c>0$ such that, for $1 \leq m \leq k+1$,

$$
\left\|D-D_{h}\right\|_{\mathbb{D}}+\left\|\mathbf{u}-\mathbf{u}_{h}\right\|_{U}+\left\|S-S_{h}\right\|_{\mathbb{S}} \leq C h^{m}\left\{\|D\|_{m}+\|\mathbf{u}\|_{m}+\|S\|_{m}+\|\operatorname{div}(S)\|_{m}\right\} .
$$




\subsection{Finite Element Subspaces for $\mathbb{D}$}

In this section a macroelement construction [33] is employed to determine subspaces $\mathbb{D}_{h} \subset \mathbb{D}$ that will satisfy the inf-sup condition (4.5) and are smaller than naive ones considered in Theorem 4.2.

\subsubsection{Macroelement Construction We employ the concept of a macroelement from [33].}

Definition 4.1 (1) A parent macroelement $\hat{M}$ is a connected finite union of simplices in $\mathbb{R}^{d}$.

(2) A macroelement $M$ in $\mathcal{T}_{h}$ affine equivalent to $\hat{M}$ is a set of simplices of $\mathcal{T}_{h}$ whose union is homeomorphic to $\hat{M}$ by a homeomorphism which is piecewise affine on each simplex $K \subset M$.

The following lemma relates local inf-sup conditions on macroelements to the corresponding condition on a parent macroelement

Lemma 4.1 Let $M$ and $\hat{M}$ be affine equivalent macroelements and let $\chi: \hat{M} \rightarrow M$ be the corresponding piecewise affine homeomorphism. If $\hat{K} \subset \hat{M}$ is a simplex and $\chi(\hat{K})=K$, write

$$
\left.\chi(\xi)\right|_{\hat{K}}=x_{0 K}+F_{K} \xi, \quad \xi \in \hat{K},
$$

where $F_{K} \in \mathbb{R}^{d \times d}$, and let $J=\operatorname{det}(F)$. Let $\hat{\mathbb{S}}(\hat{M}), \mathbb{D}_{h}(\hat{M}) \subset L^{2}(\hat{M})^{d \times d}$ and define the mappings ^: $\mathbb{S}(\hat{M}) \rightarrow \mathbb{S}_{h}(M) \subset L^{2}(M)^{d \times d}$ and $: \hat{\mathbb{D}}(\hat{M}) \rightarrow \mathbb{D}_{h}(M) \subset L^{2}(M)^{d \times d}$ by

$$
S_{h}=(1 / \sqrt{J}) F \hat{S} F^{T} \quad \text { and } \quad D_{h}=(1 / \sqrt{J}) F^{-T} \hat{D} F^{-1} .
$$

Under these transformations,

1. Symmetric matrices on $\hat{M}$ are mapped to symmetric matrices on $M$.

2. Piecewise polynomials of degree $k$ on $\hat{M}$ map to piecewise polynomials of degree $k$ on $M$.

3. If all of the Jacobian matrices $F_{K}$ are equal to a scalar multiple of an orthogonal matrix then trace free functions on $\hat{M}$ to trace free functions on $M$.

4. The mapping $\hat{\mathbb{S}}(\hat{M}) \mapsto \mathbb{S}_{h}(M)$ maps functions in $\mathbb{H}($ div, $\hat{M})$ to functions in $\mathbb{H}($ div, $M)$; moreover, divergence free functions on $\hat{M}$ map to divergence free functions on $M$. In particular, the (divergence free) Arnold-Winther space on $\hat{M}$ is mapped to the (divergence free) Arnold-Winther space on $M$.

5. For each $\hat{K} \subset \hat{M}$ and $K=\chi(\hat{K})$

$$
\left\|S_{h}\right\|_{L^{2}(K)} \leq\left\|F_{K}\right\|^{2}\|\hat{S}\|_{L^{2}(\hat{K})}, \quad\left\|D_{h}\right\|_{L^{2}(K)} \leq\left\|F_{K}^{-1}\right\|^{2}\|\hat{D}\|_{L^{2}(\hat{K})}
$$

and

$$
\int_{K_{i}} S_{h}: D_{h}=\int_{\hat{K}_{i}} \hat{S}: \hat{D}
$$

6. Define the "condition number" to be $\kappa_{M}=\|F\|_{L^{\infty}(\hat{M})}\left\|F^{-1}\right\|_{L^{\infty}(\hat{M})}$. If $\hat{S} \in \hat{\mathbb{S}} \backslash\{0\}$, then

$$
\sup _{D_{h} \in \mathbb{D}_{h}(M)} \frac{\int_{M} S_{h}: D_{h}}{\left\|D_{h}\right\|_{L^{2}(M)}\left\|S_{h}\right\|_{L^{2}(M)}} \geq \frac{1}{\kappa_{M}^{2}} \sup _{\hat{D} \in \hat{\mathbb{D}}(\hat{M})} \frac{\int_{\hat{M}}(\hat{S}, \hat{D})}{\|\hat{D}\|_{L^{2}(\hat{M})}\|\hat{S}\|_{L^{2}(\hat{M})}} .
$$

The mapping $\hat{S} \mapsto S_{h}$ is the symmetric version of the Piola transform [31,14] and the transformation $\hat{D} \mapsto D_{h}$ was constructed to be the dual operator preserving the inner product.

The next step is to show that a global inf-sup condition in $\mathcal{T}_{h}$ will follow from the local inf-sup condition on the right hand side of (4.7). 
Theorem 4.3 Let $\hat{\mathcal{M}}$ be a family of parent macroelements and $\Omega=\cup_{H} M_{H}$ be a covering of $\Omega$ by macroelements of $\mathcal{T}_{h}$ each affine equivalent to some $\hat{M} \in \hat{\mathcal{M}}$. For each $\hat{M} \in \hat{\mathcal{M}}$ let $\hat{\mathbb{D}}(\hat{M})$, $\hat{\mathbb{S}}(\hat{M}) \subset L^{2}(\hat{M})^{d \times d}$ be specified and assume that there exists $\hat{c}>0$

$$
\sup _{\hat{D} \in \hat{\mathbb{D}}(\hat{M})} \frac{\int_{\hat{M}}(\hat{S}, \hat{D})}{\|\hat{D}\|_{L^{2}(\hat{M})}} \geq \hat{c}\|\hat{S}\|_{L^{2}(\hat{M})}, \quad \hat{S} \in \hat{\mathbb{S}}(\hat{M}), \quad \hat{M} \in \hat{\mathcal{M}} .
$$

Let $\mathbb{S}_{h} \subset L^{2}(\Omega)^{d \times d}$ and assume that the local spaces $\mathbb{S}_{h}(M)$ formed by restriction to $M$ are the images of $\hat{\mathbb{S}}(\hat{M})$ under the (symmetric) Piola transform (4.6). If $\mathbb{D}_{h}$ is the (discontinuous) finite element space on $\mathcal{T}_{h}$ spanned by the local spaces $\mathbb{D}_{h}(M)$, then

$$
\sup _{D_{h} \in \mathbb{D}_{h}} \frac{\int_{\omega} S_{h}: D_{h}}{\left\|D_{h}\right\|_{L^{2}(\Omega)}} \geq \frac{\hat{c}}{\gamma \kappa^{2}}\left\|S_{h}\right\|_{L^{2}(\Omega)},
$$

where $\kappa=\max _{M} \kappa_{M}$ is the maximal condition number on each macroelement and $\gamma$ is the ply of the covering $\Omega=\cup_{H} M_{H}$; that is, $x \in \Omega$ belongs to at most $\gamma$ macroelements.

Proof Fix $S_{h} \in \mathbb{S}_{h}$ and for each $H$ let $D_{H} \in \mathbb{D}_{h}\left(M_{H}\right)$ satisfy $\left\|D_{H}\right\|_{L^{2}\left(M_{H}\right)}=\left\|S_{h}\right\|_{L^{2}\left(M_{H}\right)}$ and

$$
\int_{M_{H}} D_{H}: S_{h} \geq\left(\hat{c} / \kappa^{2}\right)\left\|S_{h}\right\|_{L^{2}\left(M_{H}\right)}^{2} .
$$

Then define $D_{h}=\sum_{H} D_{H}$, and use the property that $\left\{M_{H}\right\}$ cover $\Omega$ to conclude

$$
\int_{\Omega} D_{h}: S_{h} \geq\left(\hat{c} / \kappa^{2}\right)\left\|S_{h}\right\|_{L^{2}(\Omega)}^{2}
$$

Next, since the covering has finite ply we compute

$$
\begin{aligned}
\left\|D_{h}\right\|_{L^{2}(\Omega)}^{2} & =\sum_{K \in \mathcal{T}_{h}} \int_{K}\left|\sum_{H: K \subset M_{H}} D_{H}\right|^{2} \\
& \leq \sum_{K \in \mathcal{T}_{h}} \int_{K} \gamma \sum_{H: K \subset M_{H}}\left|D_{H}\right|^{2} \\
& =\gamma \sum_{K \in \mathcal{T}_{h}} \sum_{H: K \subset M_{H}} \int_{K}\left|D_{H}\right|^{2} \\
& =\gamma \sum_{H} \sum_{K \subset M_{H}} \int_{K}\left|D_{H}\right|^{2} \\
& =\gamma \sum_{H} \int_{M_{H}}\left|D_{H}\right|^{2} \\
& =\gamma \sum_{H} \int_{M_{H}}\left|S_{h}\right|^{2} \\
& \leq \gamma^{2} \int_{\Omega}\left|S_{h}\right|^{2} .
\end{aligned}
$$

It follows that $\left\|D_{h}\right\|_{L^{2}(\Omega)} \leq \gamma\left\|S_{h}\right\|_{L^{2}(\Omega)}$, and

$$
\frac{\int_{\Omega} D_{h}: S_{h}}{\left\|D_{h}\right\|_{L^{2}(\Omega)}} \geq\left(\hat{c} / \gamma \kappa^{2}\right)\left\|S_{h}\right\|_{L^{2}(\Omega)} .
$$


$\hat{M}$

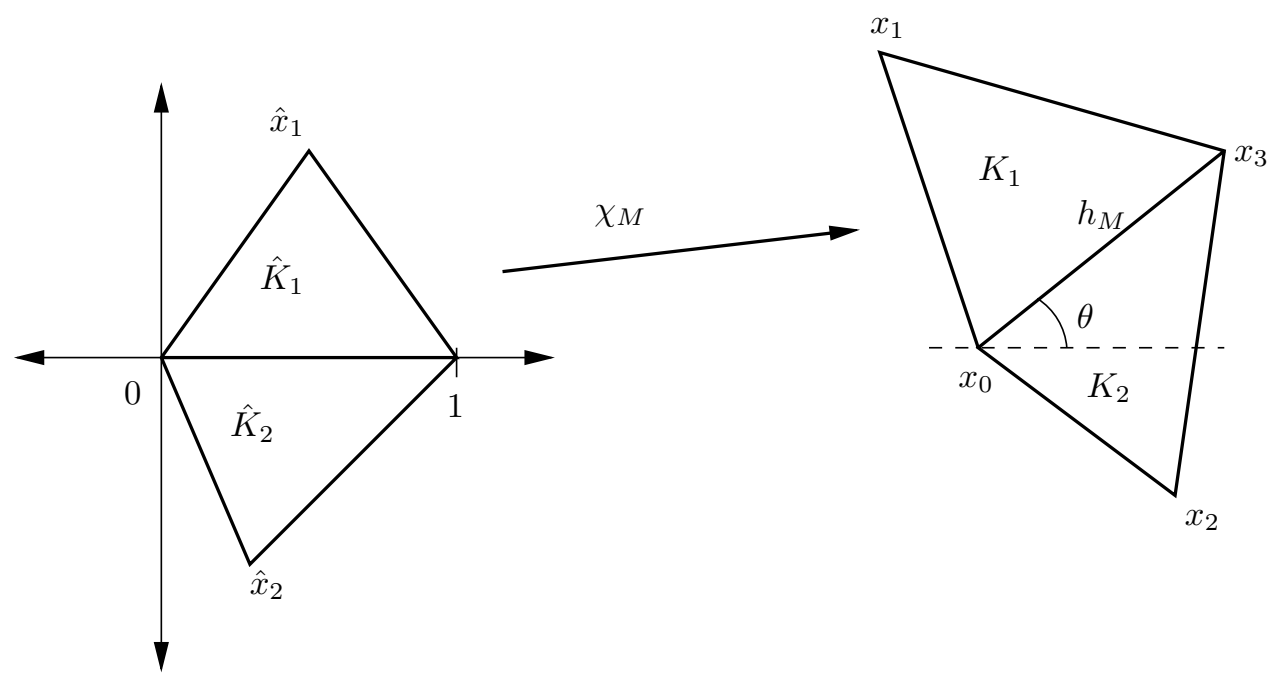

Fig. 4.2. Reference $\hat{M}$ and affine equivalent $M$ macroelements.

In practice it is trivial to construct a covering of $\Omega$ by macroelements with finite ply, and a bound upon the condition number follows from the bound upon the aspect ratio of the simplices in $\mathcal{T}_{h}$. In this situation the global inf-sup condition will follow provided the local inf-sup condition holds on each parent macroelement with constant independent of $\hat{M} \in \hat{\mathcal{M}}$. The following lemma shows that verification of the inf-sup condition on each macroelement reduces to linear algebra.

Lemma 4.2 Let $\hat{M}$ be a parent macroelement and suppose $\hat{\mathbb{D}}, \hat{\mathbb{S}} \subset L^{2}(\hat{M})_{\text {sym }}^{d \times d}$ are finite dimensional subspaces and that for all $\hat{S} \in \hat{\mathbb{S}}$

$$
\int_{\hat{M}} \hat{D}: \hat{S}=0 \quad \forall \hat{D} \in \hat{\mathbb{D}} \quad \Rightarrow \quad \hat{S}=0
$$

Then there exists $\hat{c}>0$ such that

$$
\sup _{\hat{D} \in \hat{\mathbb{D}}} \frac{\int_{\hat{M}} \hat{D}: \hat{S}}{\|\hat{D}\|_{L^{2}(\hat{M})}} \geq \hat{c}\|\hat{S}\|_{L^{2}(\hat{M})}, \quad \hat{S} \in \hat{\mathbb{S}} .
$$

Proof Let the linear map $\phi: \hat{\mathbb{D}} \rightarrow \hat{\mathbb{S}}$ be characterized by $(\phi(\hat{D}), \hat{S})_{L^{2}}=(\hat{D}, \hat{S})_{L^{2}}$ for all $\hat{S}$. The hypothesis of the theorem shows $R g(\phi)^{\perp}=\{0\}$, and finite dimensionality guarantees $R g(\phi)$ is closed so is all of $\hat{S}$. Then $\phi$ is surjective and is an isomorphism on $\operatorname{Ker}(\phi)^{\perp}$. Selecting $\hat{D}=\phi^{-1}(\hat{S}) \in$ $\operatorname{Ker}(\phi)^{\perp}$ establishes the inf-sup condition (4.8).

If $\hat{\mathcal{M}}$ is finite the inf-sup condition then reduces to linear algebra. When $\hat{\mathcal{M}}$ is infinite the macroelements can usually be parameterized by a compact set; for example, the vertices if the simplices on $\hat{M}$ typically lie in a compact set of $\mathbb{R}^{d}$. If the constants $\hat{c}=\hat{c}(\hat{M})>0$ depend continuously upon the parameters a uniform bound from below will follow (positive functions on compact sets achieve their minimum). 


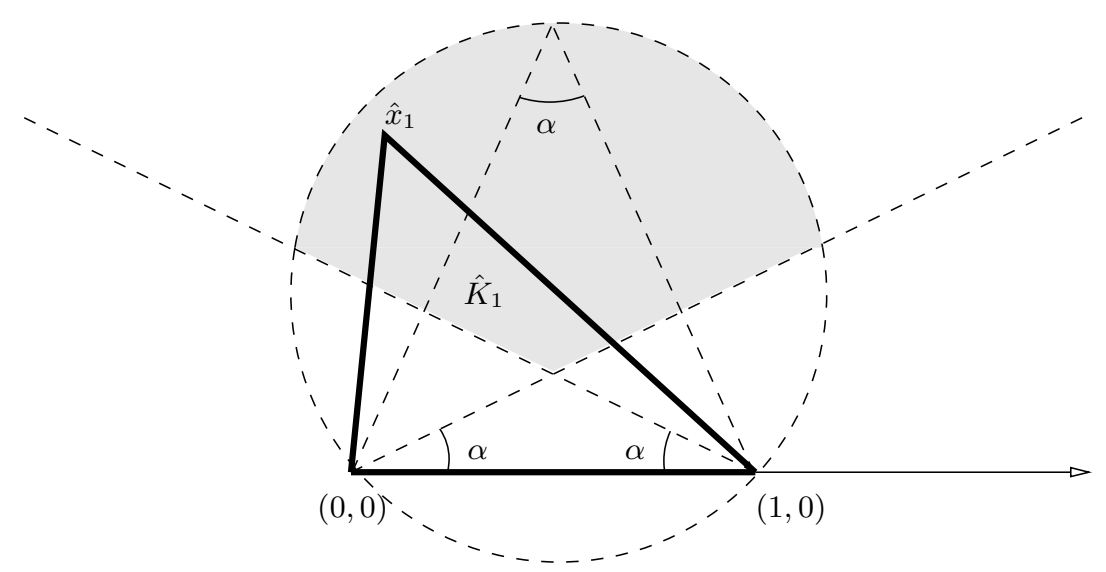

Fig. 4.3. If $\hat{K}_{1}$ has minimum angle $\alpha$ then $\hat{x}_{1}$ lies in a compact subset of $\mathbb{R}^{2}$.

4.3.2 Two Dimensional Example In this section the macroelement technique is used to show that in two dimensions the inf-sup condition (4.5) holds for the lowest order Arnold Winther elements, $k=1$, when $\mathbb{D}_{h}$ is the space of discontinuous "quadratic plus bubble" symmetric trace free matrices. For this purpose we select the macroelements to be pairs of triangles which have an edge in common, and the reference macro elements be pairs of simplices with common edge lying on the unit interval of the $x$-axis (see Figure 4.2),

$$
\hat{\mathcal{M}}=\left\{\hat{M}=\hat{K}_{1} \cup \hat{K}_{2} \mid \hat{K}_{1} \cap \hat{K}_{2}=[0,1] \times\{0\} \text {, and } \hat{K}_{1}, \hat{K}_{2} \text { have aspect ratio bounded by } C\right\} .
$$

If $\left\{\mathcal{T}_{h}\right\}$ is a regular family of triangulations of $\Omega$ then each pair of triangles $M=K_{1} \cup K_{2}$ sharing an edge of length $h_{M}$ can be mapped to $\hat{M} \in \hat{\mathcal{M}}$ with an affine homeomorphism of the form,

$$
x=\chi(\hat{x})=x_{0}+h_{M} Q \hat{x}, \quad Q^{T} Q=I .
$$

Selecting the Jacobians to be orthogonal guarantees that trace free matrices are mapped to trace free matrices under the Piola transformations (4.6), and that condition numbers $\kappa_{M}=1$ are trivially bounded.

It remains to verify the inf-sup condition on each macroelement $\hat{M} \in \hat{\mathcal{M}}$. To do this we parameterize $\hat{M}=\hat{K}_{1} \cup \hat{K}_{2} \in \hat{\mathcal{M}}$ by the coordinates of the vertices $\hat{x}_{1}$ and $\hat{x}_{2}$ of $\hat{K}_{1}$ and $\hat{K}_{2}$ not on the $\hat{x}_{1}$-axis. Since each simplex has bounded aspect ratio it follows that the pair $\left(\hat{x}_{1}, \hat{x}_{2}\right)$ lie in a compact subset of $\mathbb{R}^{2}$ as indicated in Figure 4.3. Moreover, since the inf-sup condition only involves integrals over $\hat{M}=\hat{M}\left(\hat{x}_{1}, \hat{x}_{2}\right)$ it is clear that the constant depends continuously upon the two parameters. In this situation it suffices to establish the inf-sup condition on each $\hat{M}\left(\hat{x}_{1}, \hat{x}_{2}\right)$ using, for example, Lemma 4.2 .

The linear algebra required to complete the proof of the inf-sup condition involves large matrices with symbolic components, $\left(\hat{x}_{1}, \hat{x}_{2}\right)$. Maple was used to verify the hypothesis in Lemma 4.2 as follows:

1. On each of $\hat{K}_{1}$ and $\hat{K}_{2}$ the divergence free functions in the lowest order Arnold-Winther space take the form

$$
\begin{aligned}
\hat{S}_{11}= & 1 / 2 a_{0} x^{2}+1 / 6 a_{1} x^{3}+1 / 2 a_{2} y x^{2}+\left(a_{3}+a_{4} y+a_{5} y^{2}\right) x+a_{6}+a_{7} y+a_{8} y^{2}+a_{9} y^{3} \\
\hat{S}_{12}= & -a_{0} x y-1 / 2 a_{1} x^{2} y-1 / 2 a_{2} y^{2} x-a_{3} y-1 / 2 a_{4} y^{2}-1 / 3 a_{5} y^{3} \\
& -a_{10} x-1 / 2 a_{11} x^{2}-1 / 3 a_{12} x^{3}+a_{17} \\
\hat{S}_{22}= & 1 / 2 a_{0} y^{2}+1 / 2 a_{1} x y^{2}+1 / 6 a_{2} y^{3}+\left(a_{10}+a_{11} x+a_{12} x^{2}\right) y+a_{13}+a_{14} x+a_{15} x^{2}+a_{16} x^{3}
\end{aligned}
$$


2. Accumulate the set of equations to enforce the following:

(a) $\hat{S}$ is continuous at the vertices $(0,0)$ and $(1,0)$,

$$
\hat{S}\left(0,0_{-}\right)=\hat{S}\left(0,0_{+}\right), \quad \text { and } \quad \hat{S}\left(1,0_{-}\right)=\hat{S}\left(1,0_{+}\right)
$$

(b) $\hat{S} \mathbf{n}$ is continuous across the $x$-axis where $\mathbf{n}=(0,1)^{T}$. Along the $x$-axis the normal components are univariate polynomials of degree three which agree at the end points, so it suffices to require continuity at two more points.

$$
\hat{S}\left(1 / 3,0_{-}\right) \mathbf{n}=\hat{S}\left(1 / 2,0_{+}\right) \mathbf{n}, \quad \text { and } \quad \hat{S}\left(2 / 3,0_{-}\right) \mathbf{n}=\hat{S}\left(2 / 3,0_{+}\right) \mathbf{n} .
$$

(c) On each of the triangles, $K_{i}, \hat{S}$ is orthogonal to a basis of the trace free matrices with components in $\mathcal{P}_{2}\left(\hat{K}_{i}\right) \oplus b_{\hat{K}_{i}}$.

$$
\int_{\hat{K}_{i}}\left(\hat{S}_{11}-\hat{S}_{2} 2\right) p=0, \quad \int_{\hat{K}_{i}} \hat{S}_{12} p=0, \quad p \in\left\{1, x, y, x^{2}, x y, y^{2}, b_{K_{i}}(x, y)\right\}
$$

3. Solve the system of linear equations. If the solution is a constant diagonal matrix, $\hat{S}(x, y)=\alpha I$, conclude the inf-sup condition holds. Since the coefficient matrix of the linear system has symbolic entries corresponding to the components of $\hat{x}_{1}$ and $\hat{x}_{2}$ fraction free Gauss elimination should be employed for their solution.

Granted the integrity of the linear algebra system, this procedure provides a proof of the following lemma.

Lemma 4.3 Let $\left\{\mathcal{T}_{h}\right\}_{h>0}$ be a regular family of triangulations of a bounded domain $\Omega \subset \mathbb{R}^{2}$ and let $Z_{h} \subset \mathbb{S}_{h}$ be the subspace of the lowest order $(k=1)$ Arnold-Winther tensors with divergence zero and let

$$
\mathbb{D}_{h}=\left\{D \in \mathbb{D}\left|D_{i j}\right|_{K} \in \mathbb{P}_{2}(K) \oplus b_{K} \quad \forall K \in \mathcal{T}_{h}\right\} .
$$

Then there is a constant $c>0$ independent of $h$ such that

$$
\sup _{D_{h} \in \mathbb{D}_{h}} \frac{\int_{\Omega} D_{h}: S_{h}}{\left\|D_{h}\right\|_{\mathbb{D}}} \geq c\left\|S_{h}\right\|, \quad S_{h} \in Z_{h} .
$$

The bubble component was necessary for the two element macroelement construction. However, numerical experiments suggest that it may not be required if a larger macroelement is used. For example, if $\Omega$ is a square (or L-shaped etc.) and is triangulated by dividing uniform square grids along the diagonal, then groups of 8 triangles (four squares) can be mapped to the macroelement shown in Figure 4.4(a) by maps of the form $x=\chi_{M}(\hat{x})=x_{0}+h \xi$ so that the Jacobian is $F=h I$. In this situation the linear system only contains numerical entries, and the macroelement calculations shows that the bubble is not required; that is the inf-sup condition is satisfied when

$$
\mathbb{D}_{h}=\left\{D \in \mathbb{D}\left|D_{i j}\right|_{K} \in \mathbb{P}_{2}(K) \quad \forall K \in \mathcal{T}_{h}\right\}
$$




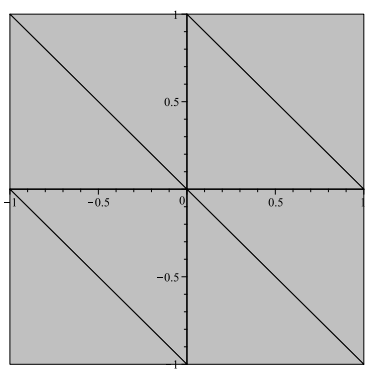

(a) Eight triangle macroelement

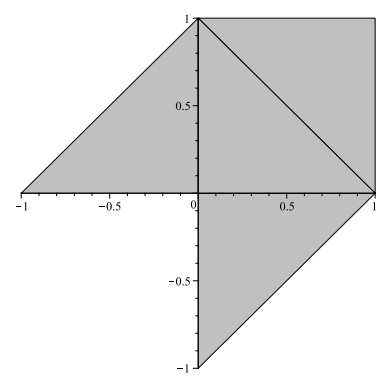

(b) Four triangle macroelement

Fig. 4.4. Parent macroelements used for inf-sup conditions for the trace-free $D$ formulation (a) and for the formulation with pressure (b).

\subsection{Alternate Formulation with Pressure}

The solution (1.6) requires

$$
\sup _{D \in \mathbb{D}} \frac{\int_{\Omega} D: S+p I: D}{\|D\|} \geq c\left(\|S\|_{\mathbb{S}}+\|p\|_{P}\right), \quad \forall(S, p) \in Z \times P
$$

The proof of (4.9) is shown by setting $D=S-(1 / e) \operatorname{tr}(S)$ (as for (4.3)) if $\|S\|_{\mathbb{S}} \geq\|p\|_{P}$ and choosing $D=p I-S \quad$ when $\|S\|_{\mathbb{S}} \leq\|p\|_{P}$.

The macroelement construction is vastly simplified in this situation since the mappings $\chi: \hat{M} \rightarrow$ $M$ do not need to preserve the trace. In particular, a single parent element suffices and the infsup condition reduces to showing a single (numerical) matrix has full rank. For the parent element with four triangles shown in Figure 4.4(b), the macroelement construction shows that the inf-sup condition (4.9) is satisfied when $\mathbb{S}_{h}$ and $U_{h}$ are as in Section 4.2, and

$$
\mathbb{D}_{h}=\left\{D \in L^{2}(\Omega)_{s y m}^{d \times d}\left|D_{i j}\right|_{K} \in \mathbb{P}_{1}(K) \oplus b_{K} \quad \forall K \in \mathcal{T}_{h}\right\}
$$

and

$$
P_{h}=\left\{p \in P|p|_{K} \in \mathbb{P}_{1}(K) \oplus b_{K} \quad \forall K \in \mathcal{T}_{h}\right\} .
$$

If $\mathcal{P}_{h}^{\mathbb{D}}$ and $\mathcal{P}_{h}^{P}$ denote the orthogonal projections, onto these spaces we have

$$
\begin{gathered}
\left\|D-\mathcal{P}_{h}^{\mathbb{D}} D\right\|_{L^{2}(\Omega)} \leq c h^{m}\|D\|_{m}, \quad 0 \leq m \leq 2 \\
\left\|p-\mathcal{P}_{h}^{P} p\right\|_{L^{2}(\Omega)} \leq c h^{m}\|p\|_{m}, \quad 0 \leq m \leq 2
\end{gathered}
$$

The error estimate for the discrete problem becomes

$$
\begin{aligned}
\left\|D-D_{h}\right\|_{\mathbb{D}}+\left\|\mathbf{u}-\mathbf{u}_{h}\right\|_{U}+\left\|S-S_{h}\right\|_{\mathbb{S}} & \left\|p-p_{h}\right\|_{P} \\
& \leq C h^{m}\left\{\|D\|_{m}+\|\mathbf{u}\|_{m}+\|S\|_{m}+\|\operatorname{div}(S)\|_{m}+\|p\|_{m}\right\} .
\end{aligned}
$$




\section{A Auxiliary Results}

Lemma A.1 Let $\Omega \subset \mathbb{R}^{d}$ be a bounded Lipschitz domain and let $\partial \Omega=\bar{\Gamma}_{0} \cup \bar{\Gamma}_{1}$ be a decomposition into two open sets with $\left|\Gamma_{1}\right| \neq 0$. Let $U=\left\{\mathbf{u} \in H^{1}(\Omega)^{d}|\mathbf{u}|_{\Gamma_{0}}=0\right\}$. Then there exists $c>0$ such that

Proof Recall that

$$
\sup _{\mathbf{u} \in U} \frac{\int_{\Omega} p \operatorname{div}(\mathbf{u})}{\|\mathbf{u}\|_{H^{1}(\Omega)}} \geq c\|p\|_{L^{2}(\Omega)}, \quad p \in L^{2}(\Omega) .
$$

$$
\sup _{\mathbf{u}_{0} \in H_{0}^{1}(\Omega)^{d}} \frac{\int_{\Omega} p \operatorname{div}\left(\mathbf{u}_{0}\right)}{\left\|\mathbf{u}_{0}\right\|_{H^{1}(\Omega)}} \geq c_{0}\|p\|_{L^{2}(\Omega) / \mathbb{R}},
$$

and $\|p\|_{L^{2}(\Omega)}^{2}=\|p\|_{L^{2}(\Omega) / \mathbb{R}}^{2}+|\Omega| \bar{p}^{2}=\|p-\bar{p}\|_{L^{2}(\Omega)}^{2}+|\Omega| \bar{p}^{2}$ where $\bar{p}$ is the average value of $p$. Let $U=H_{0}^{1}(\Omega)^{d} \oplus U_{1}$ be the orthogonal decomposition. Then writing $\mathbf{u} \in U$ as $\mathbf{u}=\mathbf{u}_{0}+\mathbf{u}_{1}$ we compute

$$
\begin{aligned}
\int_{\Omega} p \mathbf{u} & =\int_{\Omega}(p-\bar{p}) \operatorname{div}\left(\mathbf{u}_{0}+\mathbf{u}_{1}\right)+\bar{p} \operatorname{div}\left(\mathbf{u}_{1}\right) \\
& =\int_{\Omega}(p-\bar{p}) \operatorname{div}\left(\mathbf{u}_{0}+\mathbf{u}_{1}\right)+\bar{p} \int_{\Gamma_{1}} \mathbf{u}_{1} . \mathbf{n} .
\end{aligned}
$$

Since the trace operator $\gamma: U \rightarrow H^{1 / 2}\left(\Gamma_{1}\right)$ is surjective, there exists $\mathbf{u}_{1} \in U_{1}$ with $\left\|\mathbf{u}_{1}\right\|_{H^{1}(\Omega)}=1$ such that the integral on the right is positive. Then

$$
\begin{aligned}
\int_{\Omega} p \mathbf{u} & \geq \int_{\Omega}(p-\bar{p}) \operatorname{div}\left(\mathbf{u}_{0}\right)-\|p-\bar{p}\|_{L^{2}(\Omega)}+c_{1}|\bar{p}| \\
& \geq\left(c_{0}\left\|\mathbf{u}_{0}\right\|-1\right)\|p-\bar{p}\|_{L^{2}(\Omega)}+c_{1}|\bar{p}| \\
& =\left(c_{0}\left\|\mathbf{u}_{0}\right\|-1\right)\|p\|_{L^{2}(\Omega) / \mathbb{R}}+c_{1}|\bar{p}| .
\end{aligned}
$$

Selecting $\left\|\mathbf{u}_{0}\right\|=\left(1+c_{1}\right) / c_{0}$ gives $\|\mathbf{u}\| \leq C\left(c_{0}, c_{1}\right)$ which completes the proof.

Lemma A.2 Let $\Omega \subset \mathbb{R}^{d}$ be a bounded Lipschitz domain and let $\partial \Omega=\bar{\Gamma}_{0} \cup \bar{\Gamma}_{1}$ be a decomposition into two open sets with $\left|\Gamma_{1}\right| \neq 0$. Let $\mathbb{S}=\left\{S \in \mathbb{H}(\operatorname{div} ; \Omega)|S \mathbf{n}|_{\Gamma_{1}}=0\right\}$ and $U=\left\{\mathbf{u} \in H^{1}(\Omega)^{d} \mid\right.$ $\left.\left.\mathbf{u}\right|_{\Gamma_{0}}=0\right\}$. Then there exists $c>0$ such that

$$
\|\operatorname{tr}(S)\|_{L^{2}(\Omega)} \leq C\left(\left\|S_{0}\right\|_{L^{2}(\Omega)}+\|\operatorname{div}(S)\|_{U^{\prime}}\right),
$$

where $S_{0}=S-(\operatorname{tr}(S) / d) I$ is the deviatoric part of $S$.

Proof Let $(\mathbf{u}, p) \in U \times L^{2}(\Omega)$ satisfy

$$
(\mathbf{u}, \mathbf{v})_{H^{1}(\Omega)}+(p, \operatorname{div}(\mathbf{v}))_{L^{2}(\Omega)}=0, \quad(\operatorname{div}(\mathbf{u}), q)_{L^{2}(\Omega)}=(\operatorname{tr}(S), q)_{L^{2}(\Omega)},
$$

for all $(\mathbf{v}, q) \in U \times L^{2}(\Omega)$. The previous lemma shows that the inf-sup condition is satisfied, so solutions exists and $\|\mathbf{u}\|_{H^{1}(\Omega)} \leq C\|\operatorname{tr}(S)\|_{L^{2}(\Omega)}$. Then

$$
\begin{aligned}
\|\operatorname{tr}(S)\|_{L^{2}(\Omega)}^{2} & =\int_{\Omega} \operatorname{tr}(S) \operatorname{div}(\mathbf{u}) \\
& =\int_{\Omega} \operatorname{tr}(S) I:(\nabla \mathbf{u}) \\
& =d \int_{\Omega}\left(S-S_{0}\right):(\nabla \mathbf{u}) \\
& =-d \int_{\Omega} \operatorname{div}(S) \cdot \mathbf{u}+S_{0}:(\nabla \mathbf{u}) \\
& \leq d\left(\|\operatorname{div}(S)\|_{U^{\prime}}^{2}+\left\|S_{0}\right\|_{L^{2}(\Omega)}^{2}\right)^{1 / 2}\|\mathbf{u}\|_{H^{1}(\Omega)} .
\end{aligned}
$$


In the third line we used the identity $(1 / d) \operatorname{tr}(S) I=S-S_{0}$, and the fourth line follows since $S \mathbf{n} . \mathbf{u}$ vanishes on the boundary.

\section{References}

1. D. N. Arnold, G. Awanou, and R. Winther. Finite elements for symmetric tensors in three dimensions. Math. Comp., 77(263):1229-1251, 2008.

2. D. N. Arnold, F. Brezzi, and J. Douglas, Jr. PEERS: a new mixed finite element for plane elasticity. Japan J. Appl. Math., 1(2):347-367, 1984.

3. D. N. Arnold, J. Douglas, Jr., and C. P. Gupta. A family of higher order mixed finite element methods for plane elasticity. Numer. Math., 45(1):1-22, 1984.

4. D. N. Arnold, R. S. Falk, and R. Winther. Differential complexes and stability of finite element methods. I. The de Rham complex. In Compatible spatial discretizations, volume 142 of IMA Vol. Math. Appl., pages 24-46. Springer, New York, 2006.

5. D. N. Arnold, R. S. Falk, and R. Winther. Differential complexes and stability of finite element methods. II. The elasticity complex. In Compatible spatial discretizations, volume 142 of IMA Vol. Math. Appl., pages 47-67. Springer, New York, 2006.

6. D. N. Arnold, R. S. Falk, and R. Winther. Mixed finite element methods for linear elasticity with weakly imposed symmetry. Math. Comp., 76(260):1699-1723 (electronic), 2007.

7. D. N. Arnold and R. Winther. Mixed finite elements for elasticity. Numer. Math., 92(3):401-419, 2002.

8. I. Babuška. The finite element method with Lagrangian multipliers. Numer. Math., 20:179-192, $1972 / 73$.

9. J. Baranger, K. Najib, and D. Sandri. Numerical analysis of a three-fields model for a quasi-Newtonian flow. Comput. Methods Appl. Mech. Engrg., 109(3-4):281-292, 1993.

10. J. W. Barrett and W. B. Liu. Finite element error analysis of a quasi-Newtonian flow obeying the Carreau or power law. Numer. Math., 64(4):433-453, 1993.

11. J. W. Barrett and W. B. Liu. Quasi-norm error bounds for the finite element approximation of a non-Newtonian flow. Numer. Math., 68(4):437-456, 1994.

12. S. C. Brenner and L. R. Scott. The mathematical theory of finite element methods, volume 15 of Texts in Applied Mathematics. Springer-Verlag, New York, second edition, 2002.

13. F. Brezzi. On the existence, uniqueness and approximation of saddle-point problems arising from Lagrangian multipliers. Rev. Française Automat. Informat. Recherche Opérationnelle Sér. Rouge, 8(R-2):129-151, 1974.

14. F. Brezzi and M. Fortin. Mixed and hybrid finite element methods, volume 15 of Springer Series in Computational Mathematics. Springer-Verlag, New York, 1991.

15. R. Bustinza, G. N. Gatica, M. González, S. Meddahi, and E. P. Stephan. Enriched finite element subspaces for dual-dual mixed formulations in fluid mechanics and elasticity. Comput. Methods Appl. Mech. Engrg., 194(25):427-439, 2005.

16. Z. Chen. Analysis of expanded mixed methods for fourth-order elliptic problems. Numer. Methods Partial Differential Equations, 13(5):483-503, 1997.

17. Z. Chen. Expanded mixed finite element methods for linear second-order elliptic problems. I. RAIRO Modél. Math. Anal. Numér., 32(4):479-499, 1998.

18. B. Cockburn and J. Gopalakrishnan. Error analysis of variable degree mixed methods for elliptic problems via hybridization. Math. Comp., 74(252):1653-1677 (electronic), 2005.

19. V. J. Ervin, J. S. Howell, and I. Stanculescu. A dual-mixed approximation method for a three-field model of a nonlinear generalized Stokes problem. Comput. Methods Appl. Mech. Engrg., 197(33-40):2886-2900, 2008.

20. V. J. Ervin, E. W. Jenkins, and S. Sun. Coupled generalized non-linear Stokes flow with flow through a porous media. SIAM J. Numer. Anal., 47(2):929-952, 2009.

21. G. N. Gatica. Solvability and Galerkin approximations of a class of nonlinear operator equations. Z. Anal. Anwendungen, 21(3):761-781, 2002.

22. G. N. Gatica and L. F. Gatica. On the a priori and a posteriori error analysis of a two-fold saddle-point approach for nonlinear incompressible elasticity. Internat. J. Numer. Methods Engrg., 68(8):861-892, 2006.

23. G. N. Gatica, L. F. Gatica, and E. P. Stephan. A dual-mixed finite element method for nonlinear incompressible elasticity with mixed boundary conditions. Comput. Methods Appl. Mech. Engrg., 196(35-36):3348-3369, 2007.

24. G. N. Gatica, M. González, and S. Meddahi. A low-order mixed finite element method for a class of quasiNewtonian Stokes flows. I. A priori error analysis. Comput. Methods Appl. Mech. Engrg., 193(9-11):881-892, 2004.

25. G. N. Gatica, N. Heuer, and S. Meddahi. On the numerical analysis of nonlinear twofold saddle point problems. IMA J. Numer. Anal., 23(2):301-330, 2003. 
26. G. N. Gatica, A. Márquez, and S. Meddahi. A new dual-mixed finite element method for the plane linear elasticity problem with pure traction boundary conditions. Comput. Methods Appl. Mech. Engrg., 197(9-12):1115-1130, 2008.

27. G. N. Gatica and S. Meddahi. A fully discrete Galerkin scheme for a two-fold saddle point formulation of an exterior nonlinear problem. Numer. Funct. Anal. Optim., 22(7-8):885-912, 2001.

28. G. N. Gatica and F.-J. Sayas. Characterizing the inf-sup condition on product spaces. Numer. Math., 109(2):209231, 2008.

29. G. N. Gatica and E. P. Stephan. A mixed-FEM formulation for nonlinear incompressible elasticity in the plane. Numer. Methods Partial Differential Equations, 18(1):105-128, 2002.

30. V. Girault and P.-A. Raviart. Finite element methods for Navier-Stokes equations, volume 5 of Springer Series in Computational Mathematics. Springer-Verlag, Berlin, 1986. Theory and algorithms.

31. J. E. Marsden and T. J. R. Hughes. Mathematical foundations of elasticity. Prentice-Hall, Englewood Cliffs, NJ, 1983.

32. J. E. Roberts and J.-M. Thomas. Mixed and hybrid methods. In Handbook of numerical analysis, Vol. II, Handb. Numer. Anal., II, pages 523-639. North-Holland, Amsterdam, 1991.

33. R. Stenberg. Analysis of mixed finite elements methods for the Stokes problem: a unified approach. Math. Comp., 42(165):9-23, 1984. 